\title{
The Classification of Two-dimensional Nonassociative Algebras
}

\author{
Holger P. Petersson \\ To the memory of Gottfried Rüttimann
}

\begin{abstract}
We give a complete classification of two-dimensional nonassociative algebras over arbitrary base fields, including those of characteristic two.
\end{abstract}

\section{Introduction}

Let $n$ be an nonnegative integer. A solution to the classification problem for $n$-dimensional nonassociative algebras, possibly without a unit, consists in setting up a list of examples which represents each isomorphism class exactly once. Such a list may also be interpreted as a parametrization of the orbit space $\operatorname{GL}(V) \backslash \operatorname{Hom}(V \otimes V, V)$, where $V$ is an $n$-dimensional vector space acted upon canonically by the full linear group, with the induced diagonal action on $V \otimes V$ and its natural extension to $\operatorname{Hom}(V \otimes V, V)$. In this way, the classification problem for $n$-dimensional algebras relates to questions in invariant theory.

In this paper, we solve the classification problem for two-dimensional nonassociative algebras over arbitrary base fields. The principal building blocks of our classification are derived from the twodimensional split étale algebra (which agrees with the direct sum of two copies of the base field), from the theory of quadratic field extensions (including the (purely) inseparable ones in characteristic two), and from the algebra of dual numbers. In fact, our final results lead to the paradoxical conclusion that arbitrary nonassociative algebras in dimension two are almost as well understood as the (commutative-associative) ones containing a unit.

A few words about the historical context are in order. Two-dimensional real algebras which are associative were classified by Benjamin Peirce [16] as early as 1881. The classification of twodimensional real division algebras (the term "nonassociative" always being understood) is due to Althoen and Kugler [3] (see also Burdujan [5] or Cali and Josephy [6] for a similar approach). The starting point of their investigation is the fact, originally due to Segre [17], for which they provide an independent elementary proof, that a two-dimensional real division algebra contains at least one and at most three nonzero idempotents. A completely different solution to the problem was recently given by Gottschling [8]; in fact, the present work grew out of an attempt to understand Gottschling's paper. In connection with his algebraic approach to quadratic differential equations, Markus [13] classified two-dimensional real algebras, with or without zero divisors, which are commutative. Along similar lines, a quick classification of two-dimensional commutative algebras over an algebraically closed field of characteristic not 2 or 3 is due to Walcher [18]. The classification problem for arbitrary two-dimensional real algebras containing zero divisors was taken up by Luchian [12] and subsequently solved completely by Althoen and Hansen [2].

Aside from being confined to the base field of real numbers, the dominant feature most of these investigations have in common is that they invariably deal with algebras in terms of their structure 
constants and multiplication tables relative to a given basis. By contrast, the base field in our investigation is completely arbitrary, and structure constants or multiplication tables almost never play a significant role. Instead, our approach is based on the following main ingredients.

The first important step consists in setting up the dichotomy between regular and singular algebras (1.4). Using principal Albert isotopes [1, Theorem 5], Kaplansky's Trick 1.5 relates the former to unital algebras of dimension two, whose detailed description is recalled in 1.7. The key to our subsequent investigation of regular algebras is then provided by the notion of the unital heart (1.10) and the isomorphism criterion 1.12, giving necessary and sufficient conditions for two-dimensional regular algebras with the same unital heart to be isomorphic.

In Section 2, we address ourselves to the problem of classifying two-dimensional algebras whose unital heart is étale. This problem will be solved in two steps, the first one being the Tight Enumeration Theorem 2.3 which breaks up the whole class of algebras into seven disjoint blocks, the second one being the Classification Theorem 2.12 which gives necessary and sufficient conditions for any two members in each block to be isomorphic. In Section 3 we carry out the same program for two-dimensional regular algebras whose unital heart is either the algebra of dual numbers or an inseparable quadratic field extension of characteristic two. Somewhat surprisingly, the treatment of the former case (3.4), consisting of no less than twelve disjoint blocks of algebras, turns out to be much more involved than the one of the latter (3.6), with only three such blocks. Section 4 is devoted to the study of singular (= nonregular) algebras. Though this case is considerably easier to deal with than the case of regular algebras, it still takes a fair amount of work before the classification of bisingular algebras (4.11) as well as that of strictly left and right singular ones $(4.16,4.17)$ is finally complete. The paper concludes with an application in Section 5 to quasi composition algebras, i.e., to composition algebras "without a unit", whose study goes back to the author [14].

\section{Regularity and the unital heart}

1.0 Throughout this paper, we fix an arbitrary base field $k$ and a vector space $V$ over $k$. Later on, we will specify $V$ to have dimension 2 but, for the time being, $V$ is arbitrary.

1.1 The vector space of algebras. Following Koecher [11], the totality of (nonassociative, not necessarily unital) algebra structures on $V$ is denoted $\operatorname{Alg}(V)$, so

$$
\operatorname{Alg}(V)=\operatorname{Hom}_{k}\left(V \otimes_{k} V, V\right)
$$

is a vector space over $k$ in its own right, of dimension $n^{3}$ if $V$ has dimension $n \in \mathbb{N}$. Given $A \in \operatorname{Alg}(V)$, we write $x A y$ for the product of $x, y \in V$ relative to the algebra $A$ or simply $x y$ if the context is clear. The left and right multiplication of $A$ will be denoted by $L_{A}, R_{A}$, respectively, dropping subscripts whenever there is no danger of confusion. We will write $A^{\text {op }}$ for the opposite algebra of $A$, so $x A^{\mathrm{op}} y=y A x$ for all $x, y \in V$.

1.2 A group action. We write

$$
G=\mathrm{GL}(V) \times \mathrm{GL}(V)
$$

for the direct product of two copies of the full linear group of $V$ and let it act on $\operatorname{Alg}(V)$ by means of principal Albert isotopes [1, Theorem 5] as follows: Given $A \in \operatorname{Alg}(V)$ and $(f, g) \in G$, we define 
$A^{(f, g)} \in \operatorname{Alg}(V)$ by the formula

$$
x A^{(f, g)} y=f(x) A g(y) \quad(x, y \in V) .
$$

It is indeed straightforward to check that this defines a right action of $G$ on $\operatorname{Alg}(V)$ which is compatible with passing to the opposite algebra in the sense that

$$
A^{(f, g) \mathrm{op}}=A^{\mathrm{op}(g, f)} .
$$

We also have the following result.

1.3 Lemma. Let $A, B \in \operatorname{Alg}(V)$ and $(f, g) \in G$. Then every isomorphism $\varphi: A \stackrel{\sim}{\longrightarrow} B$ is also an isomorphism $\varphi: A^{(f, g)} \stackrel{\sim}{\longrightarrow} B^{\left(f^{\prime}, g^{\prime}\right)}$ where $f^{\prime}=\varphi f \varphi^{-1}, g^{\prime}=\varphi g \varphi^{-1}$.

Proof. This follows from

$$
\begin{aligned}
\varphi\left(x A^{(f, g)} y\right) & =\varphi(f(x) A g(y)) \\
& =\varphi(f(x)) B \varphi(g(y)) \\
& =f^{\prime}(\varphi(x)) B g^{\prime}(\varphi(y)) \\
& =\varphi(x) B^{\left(f^{\prime}, g^{\prime}\right)} \varphi(y)
\end{aligned}
$$

for all $x, y \in V$.

1.4 Regularity. An algebra $A \in \operatorname{Alg}(V)$ is said to be left regular if some $u \in V$ satisfies $L_{A}(u) \in$ $\mathrm{GL}(V)$; otherwise, $A$ is called left singular. Dually for the properties of being right regular and right singular. A is said to be regular if it is both left and right regular, otherwise singular. Clearly, $A$ is left regular if and only if $A^{\text {op }}$ is right regular. It also follows easily from (1.2.1) that, if $A \in \operatorname{Alg}(V)$ is regular, so is $A^{(f, g)}$ for all $(f, g) \in G$.

The importance of the preceding notions derives from the fact that regular algebras are principal Albert isotopes of unital ones. More precisely, a slight generalization due to Elduque-Pérez [7] of an old trick of Kaplansky's [10] leads to the following result.

1.5 Kaplansky's Trick. For a regular algebra $A \in \operatorname{Alg}(V)$ choose elements $u, v \in V$ such that $f=R_{A}(v), g=L_{A}(u)$ are bijective and put $B=A^{\left(f^{\prime}, g^{\prime}\right)}$ where $f^{\prime}=f^{-1}, g^{\prime}=g^{-1}$. Then $A=B^{(f, g)}$, and $B$ is unital with identity element $e=u A v$.

Proof. We have $A=B^{(f, g)}$ by 1.2 and $e=f(u)=g(v)$. Hence $u=f^{\prime}(e), v=g^{\prime}(e)$, so for all $x \in V$ we obtain

$$
e B x=f^{\prime}(e) A g^{\prime}(x)=L_{A}(u) L_{A}(u)^{-1} x=x
$$

and, similarly, $x B e=x$.

1.6 Twodimensional algebras. From now on, we focus attention on two-dimensional algebras. Accordingly, we will assume for the rest of the paper that our vector space $V$ over $k$ has dimension 2. Then every two-dimensional algebra over $k$ is isomorphic to some element of $\operatorname{Alg}(V)$.

We begin by recalling from Bourbaki [4, III $\S 2$ Proposition 3, p. 441] the following description of unital algebras in dimension two. 
1.7 Proposition. Let $K$ be a unital $k$-algebra of dimension two. Then precisely one of the following holds.

a) $K$ is étale, i.e., $K / k$ is either a separable quadratic field extension or $K \cong k \oplus k$ splits.

b) $K=k[\varepsilon], \varepsilon^{2}=0$, is the algebra of dual numbers.

c) $k$ has characteristic two, and $K / k$ is a (purely) inseparable field extension (of exponent one).

1.8 Quadratic algebras. According to 1.7, a unital $k$-algebra $K$ of dimension two is not only commutative associative but also quadratic: Referring to Bourbaki [4, III, §2, p. 440] for details, we record that there exists a unique pair $(t, n)$ consisting of a linear form $t: K \rightarrow k$, the trace, and a quadratic form $n: K \rightarrow k$, the norm, satisfying

$$
x^{2}-t(x) x+n(x) 1=0
$$

for all $x \in K$; in fact, $t(x)$ (resp. $n(x)$ ) agrees with the trace (resp. the determinant) of the linar map $L_{K}(x)$. Also, the conjugation

$$
\tau: K \longrightarrow K, x \longmapsto{ }^{\tau} x=t(x) 1-x
$$

is an involution of $K$, i.e., a $k$-automorphism of period two.

Given a unital algebra $K \in \operatorname{Alg}(V)$, we agree upon the following notational conventions: We write $x y=x K y$ for $x, y \in V$ and $L=L_{K}$ for the left (= right) multiplication of $K$. The unit group of $K$ will be denoted by $K^{\times}$, inversion by $x^{-1}$ for $x \in K^{\times}$. Recall that $x \in K$ is invertible iff $n(x) \neq 0$, and then

$$
x^{-1}=n(x)^{-1 \tau} x .
$$

We let $\operatorname{Aut}(K)$ stand for the group of $k$-algebra automorphisms of $K$. Its natural action on $K$ will be written exponentially as $(\sigma, x) \longmapsto{ }^{\sigma} x$.

In order to link two-dimensional regular algebras with unital ones in the appropriate manner, 1.5 will be important but not quite enough. We need the following additional preparation.

1.9 Lemma. Let $K \in \operatorname{Alg}(V)$ be unital and $(f, g) \in G$. Putting $K^{\prime}=K^{(f, g)}$, the following statements are equivalent.

(i) $K^{\prime}$ is unital.

(ii) $K^{\prime}$ is a Jordan isotope of $K$, i.e., there exists an element $w \in K^{\times}$satisfying $K^{\prime}=K^{(w)}$, where $K^{(w)} \in \operatorname{Alg}(V)$ is given by

$$
x K^{(w)} y=x w y
$$

for all $x, y \in V$.

(iii) $K^{\prime} \cong K$. 
In this case, $w$ in (ii) satifies $w^{-1}=1_{K^{\prime}}$ and is unique. Also, there are elements $u, v \in K^{\times}$such that $w=u v, f=L(u), g=L(v)$. Finally, $\varphi: K^{\prime} \stackrel{\sim}{\longrightarrow}$ is an isomorphism if and only if $\varphi=\sigma L(w)$ for some $\sigma \in \operatorname{Aut}(K)$.

Proof. The implication (iii) $\Rightarrow$ (i) is obvious.

(i) $\Rightarrow$ (ii). By (1.2.1), the identity element $e^{\prime}$ of $K^{\prime}$ fulfills the relations

$$
L\left(f\left(e^{\prime}\right)\right) g=\mathbf{1}=R\left(g\left(e^{\prime}\right)\right) f=L\left(g\left(e^{\prime}\right)\right) f,
$$

so $f\left(e^{\prime}\right), g\left(e^{\prime}\right)$ are invertible in $K$, with inverses $u=g\left(e^{\prime}\right)^{-1}, v=f\left(e^{\prime}\right)^{-1}$ satisfying $f=L(u), g=$ $L(v)$. Hence, setting $w=u v$,

$$
x K^{\prime} y=f(x) g(y)=u x v y=x w y
$$

for all $x, y \in V$, and this is (ii).

(ii) $\Rightarrow$ (iii). One checks immediately that $L(w): K^{\prime}=K^{(w)} \stackrel{\sim}{\longrightarrow} K$ is an ismorphism, giving (iii).

Of the additional statements, the first one is obvious whereas the second one has been established in the course of proving the implication (i) $\Rightarrow$ (ii). Finally, $L(w)$ trivially being an isomorphism $K^{\prime} \stackrel{\sim}{\longrightarrow} K$, an arbitrary map from $K^{\prime}$ to $K$ is an isomorphism if and only if it differs from $L(w)$ by an automorphism of $K$ as indicated.

1.10 Unital Heart Proposition. For all $A \in \operatorname{Alg}(V)$, the following statements are equivalent.

(i) A is regular.

(ii) There exist a unital algebra $K \in \operatorname{Alg}(V)$ and $(f, g) \in G$ satisfying $A \cong K^{(f, g)}$.

In this case, $K$ is unique up to isomorphism and is called the unital heart of $A$.

Proof. While the implication (i) $\Rightarrow$ (ii) has been established in 1.5, its converse was noted in 1.4. It therefore remains to show that $K$ as in (ii) is unique up to isomorphism. To see this, let also $K^{\prime} \in \operatorname{Alg}(V)$ be unital such that $A \cong K^{\prime\left(f^{\prime}, g^{\prime}\right)}$ for some $\left(f^{\prime}, g^{\prime}\right) \in G$. Then there are $\left(f^{\prime \prime}, g^{\prime \prime}\right) \in G$ satisfying $K^{\prime} \cong K^{\left(f^{\prime \prime}, g^{\prime \prime}\right)}(1.2,1.3)$, forcing $K^{\prime}$ and $K$ to be isomophic (1.9).

1.11 Classification strategy. Before we proceed to outline our strategy for solving the classification problem, a word of caution about 1.7. It would be a mistake to construe this result as yielding a classification of unital algebras in dimension two since the structure of quadratic field extensions remains completely in the dark and actually depends on $k$ itself in a delicate manner; for example, separable quadratic field extensions are classified by $H^{1}(k, \mathbb{Z} / \mathbb{Z} 2)$.

On the other hand, our principal results will show that arbitrary two-dimensional algebras are fully understood once a full understanding of quadratic field extensions, not necessarily separable, is taken for granted. An essential part of this will be accomplished by fixing an arbitrary unital $k$-algebra $K$ in dimension two and by classifying all two-dimensional regular algebras whose unital heart is $K$. As we shall see below, the outcome of this classification strongly depends on which type a),b),c) in 1.7 the algebra $K$ belongs to. In addition, the following isomorphism criterion, which happens to generalize 1.9 , will play a crucial role. 
1.12 Isomorphism Criterion. Let $K \in \operatorname{Alg}(V)$ be unital and $(f, g),\left(f^{\prime}, g^{\prime}\right) \in G$. Then for any $\varphi$ the following statements are equivalent.

(i) $\varphi: K^{(f, g)} \stackrel{\sim}{\longrightarrow} K^{\left(f^{\prime}, g^{\prime}\right)}$ is an isomorphism.

(ii) There are elements $u, v \in K^{\times}$and $\sigma \in \operatorname{Aut}(K)$ satisfying

$$
\begin{aligned}
& f^{\prime}=L\left(v^{-1}\right) \sigma f \sigma^{-1} L(u v), \\
& g^{\prime}=L\left(u^{-1}\right) \sigma g \sigma^{-1} L(u v), \\
& \varphi=L\left((u v)^{-1}\right) \sigma .
\end{aligned}
$$

Proof. (i) $\Rightarrow$ (ii). By 1.2, 1.3 we obtain an isomorphism

$$
\varphi: K \stackrel{\sim}{\longrightarrow} K^{\left(f^{\prime \prime}, g^{\prime \prime}\right)}, f^{\prime \prime}=f^{\prime} \varphi f^{-1} \varphi^{-1}, g^{\prime \prime}=g^{\prime} \varphi g^{-1} \varphi^{-1},
$$

so 1.9 applies to the isomorphism $\psi=\varphi^{-1}: K^{\left(f^{\prime \prime}, g^{\prime \prime}\right)} \stackrel{\sim}{\longrightarrow}$. Hence we can find invertible elements $u, v \in K$ and $\rho \in \operatorname{Aut}(K)$ such that $f^{\prime \prime}=L(u), g^{\prime \prime}=L(v), \psi=\rho L(u v)$. Setting $\sigma=\rho^{-1}$, this implies $\varphi=L\left((u v)^{-1}\right) \sigma$,

$$
\begin{aligned}
f^{\prime} & =f^{\prime \prime} \varphi f \varphi^{-1}=L(u) L\left((u v)^{-1}\right) \sigma f \sigma^{-1} L(u v) \\
& =L\left(v^{-1}\right) \sigma f \sigma^{-1} L(u v)
\end{aligned}
$$

and, similarly, $g^{\prime}=L\left(u^{-1}\right) \sigma g \sigma^{-1} L(u v)$.

(ii) $\Rightarrow$ (i). Observing $f^{\prime}=L\left(v^{-1}\right) \sigma f \varphi^{-1}, g^{\prime}=L\left(u^{-1}\right) \sigma g \varphi^{-1}$, we compute, for all $x, y \in V$,

$$
\begin{aligned}
\varphi(x) K^{\left(f^{\prime}, g^{\prime}\right)} \varphi(y) & =f^{\prime}(\varphi(x)) g^{\prime}(\varphi(y)) \\
& =v^{-1} \sigma(f(x)) u^{-1} \sigma(g(y)) \\
& =(u v)^{-1} \sigma(f(x) g(y)) \\
& =\varphi\left(x K^{(f, g)} y\right) .
\end{aligned}
$$

Hence $\varphi: K^{(f, g)} \stackrel{\sim}{\longrightarrow} K^{\left(f^{\prime}, g^{\prime}\right)}$ is an isomorphism.

1.13 Corollary. Let $K \in \operatorname{Alg}(V)$ be unital and $(f, g) \in G$. Then

$\left(\operatorname{det} f \bmod n\left(K^{\times}\right), \operatorname{det} g \bmod n\left(K^{\times}\right)\right)$

is an invariant of $K^{(f, g)}$ in $\left(k^{\times} / n\left(K^{\times}\right)\right)^{2}$.

Proof. Compare determinants in 1.12 (ii) and use $n(x)=\operatorname{det} L(x)$ for $x \in V$ (1.8).

1.14 Corollary. In 1.13 we have

$$
K^{(f, g)} \cong K^{(a f, b g)}
$$

for all $a, b \in k^{\times}$.

Proof. Put $u=a 1, v=b 1, \sigma=\mathbf{1}$ in 1.12 . 


\section{Regular Algebras with étale heart}

2.0 In this section, we fix an étale $k$-algebra $K \in \mathrm{Alg}(V)$ and wish to classify all two-dimensional regular algebras with unital heart isomorphic to $K$. For this purpose, we add to 1.8 the following notational conventions. Writing

$$
S(K)=\{x \in V \mid n(x)=1\}
$$

for the "unit sphere" belonging to the quadratic space $(V, n)$, we have

$$
S(K)=\left\{{ }^{\tau} v v^{-1} \mid v \in K^{\times}\right\}
$$

by Hilbert's Theorem 90. Once and for all we choose a full set of representatives containing 1 of $K^{\times} \bmod S(K)$ and call it $M$. Also, if $K \cong k \oplus k$ is split, we pick one of the two idempotents $\neq 0,1$ of $K$ and call it $c$. Notice that

$$
\operatorname{Aut}(K)=\{\mathbf{1}, \tau\}
$$

2.1 The Cayley-Dickson doubling process. Starting from $K$ and a scalar $a \in k^{\times}$, the CayleyDickson doubling process leads to a quaternion algebra Cay $(K, a)$ over $k$ which is defined on $K \oplus K$ by the multiplication

$$
(x, y)\left(x^{\prime}, y^{\prime}\right)=\left(x y+a^{\tau} y^{\prime} y, y^{\prime} x+y^{\tau} x^{\prime}\right)
$$

for $x, y, x^{\prime}, y^{\prime} \in K$, see [15] for details. The key to the classification of algebras with étale heart is the following elementary observation for which I have not found any reference in the literature.

2.2 Proposition. The assignment

$$
(x, y) \longmapsto L(x)+L(y) \tau
$$

gives an isomorphism $\operatorname{Cay}(K, 1) \stackrel{\sim}{\longrightarrow} \operatorname{End}_{k}(V)$ of quaternion algebras. In particular, we have

$$
\operatorname{End}_{k}(V)=L(K) \oplus L(K) \tau
$$

as a direct sum of subspaces and

$$
\operatorname{det}(L(x)+L(y) \tau)=n(x)-n(y)
$$

for all $x, y \in V$.

Proof. Since we are dealing with simple algebras of the same dimension, the first part will follow once we have shown that the map in question is a homomorphism. But this is an immediate consequence of (2.1.1) combined with the relation $\tau L(y)=L\left({ }^{\tau} y\right) \tau$ for all $y \in K$. The second statement is now obvious whereas the third follows from the fact that norms of composition algebras are preserved by isomorphisms.

2.3 Tight Enumeration Theorem for Algebras with Étale Heart. Let $A$ be a regular $k$ algebra of dimension two with unital heart isomorphic to a given two-dimensional étale $k$-algebra $K$. Then $A$ is isomorphic to precisely one of the following.

a) $K^{(1+L(y) \tau, g)}, y \in M-\{1\}, g \in \mathrm{GL}(V)$. 
b) $K^{(\mathbf{1}, \tau)}$.

c) $K^{(\tau, L(y) \tau)}, y \in S(K)$.

d) $K^{(\rho, \mathbf{1}+L(y) \tau)}, \rho \in\{\mathbf{1}, \tau\}, y \in K, n(y) \neq 1$.

e) $K^{(\rho, L(c)+L(y) \tau)}, K$ split $, \rho \in\{\mathbf{1}, \tau\}, y \in K^{\times}, c y=c$.

f) $K^{(\mathbf{1}+L(c) \tau, g)}, K$ split, $g \in \mathrm{GL}(V)$,

g) $K^{(L(c)+\tau, g)}, K$ split, $g \in \mathrm{GL}(V)$.

2.4 The proof of the Tight Enumeration Theorem requires several steps. We begin by introducing the additional notation

$$
\operatorname{Id}(K)=\left\{d \in K \mid d^{2}=d\right\}
$$

for the set of idempotents in $K$. Notice that $\operatorname{Id}(K)$ consists of four or two elements according as $K$ is split or not. Given $d \in \operatorname{Id}(K)$ we put $d^{*}=1-d \in \operatorname{Id}(K)$. For $d \neq 0,1$, which can only happen if $K$ is split, we have

$$
n\left(a d+b d^{*}\right)=a b
$$

for $a, b \in k$.

2.5 Lemma. Every element $x \in K$ admits an element $v \in K^{\times}$satisfying $v^{-1} x \in \operatorname{Id}(K)$.

Proof. For $x \in K^{\times}$(resp. $\left.x=0\right)$ put $v=x$ (resp. $v=1$ ). We are left with the case $0 \neq x \in K-K^{\times}$. Then there are $d \in \operatorname{Id}(K)$ and $a \in k^{\times}$such that $d \neq 0,1$ and $x=a d$. Putting $v=a d+d^{*}$, we are done.

2.6 Lemma. For all $y, y^{\prime} \in K$ satisfying $n(y)=n\left(y^{\prime}\right)$, the following statements are equivalent.

(i) There exists an element $w \in K$ such that $n(w)=1$ and $y^{\prime}=w y$.

(ii) $y, y^{\prime}$ are both invertible or there exists an element $a \in k^{\times}$such that $y^{\prime}=a y$.

Proof. The assertion is obvious if $y, y^{\prime}$ are both invertible or $y=0$ or $y^{\prime}=0$. By symmetry we are left with the case $0 \neq y \in K-K^{\times}$. Then there are elements $d \in \operatorname{Id}(K), b \in k^{\times}$such that $d \neq 0,1$ and $y=b d$.

(i) $\Longrightarrow$ (ii). We have $w=a d+a^{-1} d^{*}$ for some $a \in k^{\times}((2.4 .2))$, hence $y^{\prime}=w y=a b d=a y$.

(ii) $\Longrightarrow$ (i). $y^{\prime}=a y=w y$ where $w=a d+a^{-1} d^{*} \in k$ has norm 1 ((2.4.2)).

2.7 Lemma. Let $d \in \operatorname{Id}(K), d \neq 0,1$.

a) For every $a \in k$ there is an element $u \in K$ satisfying $n(u)=a$ and $d u=d$.

b) If $v \in K$ and $\sigma \in \operatorname{Aut}(K)$ satisfy $d v={ }^{\sigma} d$, then $\sigma=\mathbf{1}$.

c) If $v \in K$ satisfies $d v=d^{\tau} v$, then $v=a 1$ for some $a \in k$.

Proof. a) Put $u=d+a d^{*}$ and observe (2.4.2).

b) This ist clear since $d$ and ${ }^{\tau} d=d^{*}$ generate complementary ideals in $K$.

c) Applying $\tau$ to the relation $d v=d^{\tau} v$ yields $d^{*} v=d^{*} v$, and adding up shows that $v$ remains fixed under $\tau$. 
2.8 Lemma. Let $d, d^{\prime} \in \operatorname{Id}(K), g, g^{\prime} \in \mathrm{GL}(V)$, and suppose $y, y^{\prime} \in K$ satisfy $n(d) \neq n(y), n\left(d^{\prime}\right) \neq$ $n\left(y^{\prime}\right)$.

a) We have

$$
K^{(L(d)+L(y) \tau, g)} \cong K^{\left(L\left(d^{\prime}\right)+L\left(y^{\prime}\right) \tau, g^{\prime}\right)}
$$

if and only if there are elements $u, v \in K^{\times}$and $\sigma \in \operatorname{Aut}(K)$ such that

$$
d^{\prime}=u^{\sigma} d, y^{\prime}={ }^{\tau} u^{\tau} v v^{-1 \sigma} y
$$

and

$$
g^{\prime}=L\left(u^{-1}\right) \sigma g \sigma^{-1} L(u v) .
$$

b) If the statements of a) are fulfilled, then

$$
d^{\prime}={ }^{\sigma} d
$$

and $y^{\prime}$ is invertible (resp. nonzero) if and only if $y$ is. Finally, $d=1$ forces $d^{\prime}=u=1$.

Proof. By the isomorphism criterion 1.12, the two algebras are isomorphic if and only if $u, v \in$ $K^{\times}, \sigma \in \operatorname{Aut}(K)$ exist such that $g^{\prime}=L\left(u^{-1}\right) \sigma g \sigma^{-1} L(u v)$ and

$$
\begin{aligned}
L\left(d^{\prime}\right)+L\left(y^{\prime}\right) \tau & =L\left(v^{-1}\right) \sigma(L(d)+L(y) \tau) \sigma^{-1} L(u v) \\
& =L\left(v^{-1}\right)\left(L\left({ }^{\sigma} d\right)+L\left({ }^{\sigma} y\right) \tau\right) L(u v) \\
& =L\left(u^{\sigma} d\right)+L\left({ }^{\tau} u^{\tau} v v^{-1 \sigma} y\right) \tau
\end{aligned}
$$

Comparing components in the sense of (2.2.1), the asserted equivalence follows. If both statements are fulfilled,

$$
u^{\sigma} d=d^{\prime}=d^{\prime 2}=u^{2 \sigma} d^{2}=u^{2 \sigma} d,
$$

forcing $d^{\prime}=u^{\sigma} d={ }^{\sigma} d$, as claimed. The rest is clear.

2.9 Lemma. Let $(f, g) \in G$. Then there exists an element $\left(f^{\prime}, g^{\prime}\right) \in G$ such that $K^{(f, g)} \cong K^{\left(f^{\prime}, g^{\prime}\right)}$ and $f^{\prime}$ satisfies one of the following conditions.

a) $f^{\prime}=\mathbf{1}+L(y) \tau$ for some $y \in K^{\times}, n(y) \neq 1$.

b) $f^{\prime}=\mathbf{1}+L(d) \tau$ for some $d \in \operatorname{Id}(K), d \neq 1$.

c) $f^{\prime}=L(d)+\tau$ for some $d \in \operatorname{Id}(K), d \neq 1$.

Proof. By 2.2 we have $f=L(x)+L(y) \tau$ for some $x, y \in V$ satisfying $n(x) \neq n(y)$. Hence 2.5 yields an element $v \in K^{\times}$such that $e=v^{-1} x \in \operatorname{Id}(K)$. Applying the isomorphism criterion 1.12 with $u=v^{-1}, \sigma=1$, we may assume $x=e \in \operatorname{Id}(K)$ and are then left with the following cases.

Case 1. $e=1$.

For $y \in K^{\times}$we obtain a). For $y \in K-K^{\times}$we obtain $y=a d$ for some $a \in k^{\times}, d \in \operatorname{Id}(K)-\{1\}$. Since $n(y)=0=n(d)$, we conclude from 2.6 that $d=w y$ for some $w \in K$ having norm 1 . Hence there exists $v \in K^{\times}$such that $w={ }^{\tau} v v^{-1}((2.0 .2))$, so 2.8 a) for $u=1, \sigma=1$ leads to some $f^{\prime}$ as in b). 
Case 2. $e=0$.

Then $y \in K^{\times}$, and 2.8 a) for $u={ }^{\tau} y^{-1}, \sigma=\mathbf{1}, v=1$ leads to some $f^{\prime}$ as in c) (with $d=0$ ).

Case 3. $e \neq 0,1$.

Then $n(y) \neq n(e)=0$, so $y$ is invertible, and 2.7 a) yields an element $u \in K$ satisfying $u e=e$ and $n(u)=n\left(y^{-1}\right)$. This implies $n\left({ }^{\tau} u y\right)=1$, hence ${ }^{\tau} u y={ }^{\tau} w w^{-1}$ for some $w \in K^{\times}((2.0 .2))$. We may therefore apply 2.8 a) with $v=w^{-1}, \sigma=\mathbf{1}$ to arrive at some $f^{\prime} \in \operatorname{GL}(V)$ as in c).

2.10 We are now in a position to carry out the proof of 2.3. The Unital Heart Proposition 1.10 allows us to assume $A=K^{(f, g)}$ for some $(f, g) \in G$, and by 2.9 we are left with the following cases.

Case 1. $f=\mathbf{1}+L(y) \tau, y \in K^{\times}, n(y) \neq 1$.

Then $M$ contains an element $y^{\prime} \neq 1$ satisfying $n(y)=n\left(y^{\prime}\right)$, so some $v \in K^{\times}$has $y^{\prime}={ }^{\tau} v v^{-1} y$ $((2.0 .2))$. Setting $u=1, \sigma=\mathbf{1}$ in 2.8 , we conclude that there exists a $g^{\prime} \in \mathrm{GL}(V)$ such that $A \cong K^{\left(f^{\prime}, g^{\prime}\right)}$ where $f^{\prime}=\mathbf{1}+L\left(y^{\prime}\right) \tau$. Hence $A$ satisfies 2.3 a).

Case 2. $f=\mathbf{1}+L(d) \tau, d \in \operatorname{Id}(K), d \neq 1$.

We consider the following subcases.

Case 2.1. $d=0$.

Then $f=\mathbf{1}$ and $g=L(x)+L(y) \tau$ where $x, y \in V$ satisfy $n(x) \neq n(y)(2.2)$. Choosing a unit $v \in K$ satisfying $e=v x \in \operatorname{Id}(K)(2.5)$, we may apply 2.8 with $u=1, v, \sigma=\mathbf{1}$ to conclude $A \cong K^{\left(\mathbf{1}, g^{\prime}\right)}$ where $g^{\prime}=L(e)+L\left(y^{\prime}\right) \tau$ for some $y^{\prime} \in K$. Hence we may assume $g=L(e)+L(y) \tau$ such that $e \in \operatorname{Id}(K), y \in V$ satisfy $n(e) \neq n(y)$. This being so, we break the situation up into the following sub-subcases.

Case 2.1.1. $e=1$.

Then $g=\mathbf{1}+L(y) \tau$ and $n(y) \neq 1$. Hence $A$ satisfies $2.3 \mathrm{~d}$ ) with $\rho=\mathbf{1}$.

Case 2.1.2. $e=0$.

Then $g=L(y) \tau$, and $y$ is invertible. Setting $u=1, v={ }^{\tau} y^{-1}, \sigma=1$ in 2.8 , we conclude that $A \cong K^{(\mathbf{1}, \tau)}$ satisfies $2.3 \mathrm{~b}$ ).

Case 2.1.3. $e \neq 0,1$.

Then $K$ is split, and $y$ is invertible. Also, there are $\sigma \in \operatorname{Aut}(K)$ and $a \in k^{\times}$satisfying $c=\sigma_{e}$ and $e y=a e$. Setting $u=1, v=c+a^{-1} c^{*}$ in 2.8 , we conclude $A \cong K^{\left(\mathbf{1}, g^{\prime}\right)}$ where

$$
\begin{aligned}
g^{\prime} & =\sigma(L(e)+L(y) \tau) \sigma^{-1} L(v) \\
& =L(c v)+L\left({ }^{\sigma} y^{\tau} v\right) \tau \\
& =L(c)+L(z) \tau
\end{aligned}
$$

and $z={ }^{\sigma} y^{\tau} v$ has $c z=c^{\sigma} y^{\tau} v=a c^{\tau} v=c$. Hence $A$ satisfies 2.3 e) with $\rho=\mathbf{1}$.

Case 2.2. $d \neq 0,1$.

Then $K$ is split and $c={ }^{\sigma} d$ for some $\sigma \in \operatorname{Aut}(K)$. Also, setting $u=v=1$ in 2.8, we conclude $A \cong K^{\left(f^{\prime}, g^{\prime}\right)}$ for $f^{\prime}=\mathbf{1}+L(c) \tau$ and some $g^{\prime} \in \mathrm{GL}(V)$. Hence $A$ satisfies $\left.2.3 \mathrm{f}\right)$.

Case 3. $f=L(d)+\tau, d \in \operatorname{Id}(K), d \neq 1$.

Again we consider the following subcases.

Case 3.1. $d=0$.

Then $f=\tau$ and $g=L(x)+L(y) \tau$ where $x, y \in V$ satisfy $n(x) \neq n(y)$. By 2.5 we find a unit $w \in K$ 
such that $e=w^{-1} x \in \operatorname{Id}(K)$. Now 2.8 with $v=w^{-1}, u=v^{-1 \tau} v, \sigma=\mathbf{1}$ implies $A \cong K^{\left(f^{\prime}, g^{\prime}\right)}$ where $f^{\prime}=\tau$ and

$$
\begin{aligned}
g^{\prime} & =L\left(v^{\tau} v^{-1}\right)(L(x)+L(y) \tau) L\left({ }^{\tau} v\right) \\
& =L(e)+L\left(v^{2} v^{-1} y\right) \tau
\end{aligned}
$$

Hence we may assume $g=L(e)+L(y) \tau$ such that $e \in \operatorname{Id}(K), y \in V$ satisfy $n(e) \neq n(y)$. As before, we proceed by considering the following sub-subcases.

Case 3.1.1. $e=1$.

This gives $g=\mathbf{1}+L(y) \tau$ and $n(y) \neq 1$, so $A$ satisfies $2.3 \mathrm{~d}$ ) with $\rho=\tau$.

Case 3.1.2. $e=0$.

Then $g=L(y) \tau$ and $y \in K^{\times}$. Hence 2.8 with $v=y^{-1}, u={ }^{\tau} v v^{-1}={ }^{\tau} y^{-1} y, \sigma=1$ yields $A \cong K^{\left(\tau, g^{\prime}\right)}$ where

$$
g^{\prime}=L\left({ }^{\tau} y y^{-1}\right) L(y) \tau L\left({ }^{\tau} y^{-1}\right)=L(z) \tau
$$

and $z={ }^{\tau} y y^{-1}$ has norm 1 . Therefore $A$ satisfies $2.3 \mathrm{c}$ ).

Case 3.1.3. $e \neq 0,1$.

Then $K$ is split and $y$ is invertible. Also, there are $\sigma \in \operatorname{Aut}(K)$ and $a \in k^{\times}$satisfying $c={ }^{\sigma} e$ and $e y=a e$. Setting $v=c+a c^{*}, u={ }^{\tau} v v^{-1}$ in 2.8 , we obtain $A \cong K^{\left(\tau, g^{\prime}\right)}$ where

$$
\begin{aligned}
g^{\prime} & =L\left({ }^{\tau} v^{-1} v\right) \sigma(L(e)+L(y) \tau) \sigma^{-1} L\left({ }^{\tau} v\right) \\
& =L(v c)+L\left({ }^{\tau} v^{-1} v^{2} \sigma\right) \tau \\
& =L(c)+L(z) \tau
\end{aligned}
$$

and $z={ }^{\tau} v^{-1} v^{2 \sigma} y$ satisfies

$$
\begin{aligned}
c z & ={ }^{\tau}{ }^{-1} v^{2} c^{\sigma} y={ }^{\tau} v^{-1} v^{2} a c \\
& =a c\left(a^{-1} c+c^{*}\right)\left(c+a^{2} c^{*}\right)=c .
\end{aligned}
$$

Hence $A$ satisfies 2.3 e) with $\rho=\tau$.

Case 3.2. $d \neq 0,1$.

Then $c={ }^{\sigma} d$ for some $\sigma \in \operatorname{Aut}(K)$, and 2.8 with $u=v=1$ yields an element $g^{\prime} \in \mathrm{GL}(V)$ such that $A \cong K^{\left(f^{\prime}, g^{\prime}\right)}$ where $f^{\prime}=L(c)+\tau$. Hence $A$ satisfies $\left.2.3 \mathrm{~g}\right)$.

To complete the proof of 2.3 , it now suffices to show that algebras $A, B$ belonging to different ones among the types a) - g) cannot be isomorphic. But this follows immediately by observing (1.2.2) and by applying 2.8 either to $A, B$ or to $A^{\text {op }}, B^{\text {op }}$.

2.11 In order to complete the classification of algebras with étale heart, it is obviously enough to exhibit necessary and sufficient conditions for two algebras belonging to the same type a) - $g$ ) of the Tight Enumeration Theorem 2.3 to be isomorphic. This objective is empty for type b) and for the remaining types will be accomplished as follows. 
2.12 Classification Theorem for Algebras with Étale Heart. Let $K$ be a two-dimensional étale $k$-algebra as in 2.0 .

a) For $y, z \in M-\{1\}$ and $g, h \in \mathrm{GL}(V)$, we have

$$
K^{(\mathbf{1}+L(y) \tau, g)} \cong K^{(\mathbf{1}+L(z) \tau, h)}
$$

if and only if $y=z$ and there exists an element $a \in k^{\times}$such that $h=a g$ or $h=a \tau g \tau L\left(y^{-1}\right)$.

c) For $y, z \in S(K)$ we have

$$
K^{(\tau, L(y) \tau)} \cong K^{(\tau, L(z) \tau)}
$$

if and only if $y \equiv z \bmod S(K)^{3}$ or $y \equiv \tau_{z} \bmod S(K)^{3}$.

d) For $\rho, \sigma \in\{\mathbf{1}, \tau\}$ and $y, z \in K$ satisfying $n(y) \neq 1 \neq n(z)$, we have

$$
K^{(\rho, \mathbf{1}+L(y) \tau)} \cong K^{(\sigma, \mathbf{1}+L(z) \tau)}
$$

if and only if $\rho=\sigma$ and either $y=z$ or $y=\tau_{z}$.

e) Let $K$ be split. For $\rho, \sigma \in\{\mathbf{1}, \tau\}$ and $y, z \in K^{\times}$satisfying $c y=c=c z$, we have

$$
K^{(\rho, L(c)+L(y) \tau)} \cong K^{(\sigma, L(c)+L(z) \tau)}
$$

if and only if $\rho=\sigma$ and $y=z$.

f) Let $K$ be split. For $g, h \in \mathrm{GL}(V)$ we have

$$
K^{(\mathbf{1}+L(c) \tau, g)} \cong K^{(\mathbf{1}+L(c) \tau, h)}
$$

if and only if there exists an element $a \in k^{\times}$such that $h=a g$.

g) Let $K$ be split. For $g, h \in \mathrm{GL}(V)$ we have

$$
K^{(L(c)+\tau, g)} \cong K^{(L(c)+\tau, h)}
$$

if and only if there exists an element $a \in k^{\times}$satisfying $h=a g$.

Proof. The two algebras mentioned in each one of the six cases a), c) - g) above are isomorphic if and only if $u, v \in K^{\times}$and $\iota \in \operatorname{Aut}(K)$ exist such that the relations stated in 2.8 a), henceforth referred to as critical, are fulfilled. It therefore suffices to determine in each case what these critical relations mean for the parameters involved.

a) Here the critical relations read

$$
z={ }^{\tau} v v^{-1 \sigma} y, h=\iota g \iota^{-1} L(v)
$$

and imply $n(z)=n(y)$, hence $y=z$ by the definition of $M(2.0)$. Therefore (1) holds if and only if there exists a scalar $a \in k^{\times}$such that $v=a 1, h=a g$ for $\iota=\mathbf{1}$ and $v=a y^{-1}, h=a \tau g \tau L\left(y^{-1}\right)$ for $\iota=\tau$.

c) The critical relations have the form

$$
1={ }^{\tau} u^{\tau} v v^{-1}, L(z) \tau=L\left(u^{-1}\right) \iota L(y) \tau \iota^{-1} L(u v),
$$


allowing us to deduce first $u={ }^{\tau} v v^{-1}$ and then $z={ }^{\tau} v^{-1} v^{2} \iota$. Comparing norms, we conclude $v \in S(K)$, hence $z=v^{3 \iota} y$. Reading this argument backwards completes the proof of c).

d) By $2.8 \mathrm{~b}$ ) we may assume $\rho=\sigma$ and then distinguish the following cases.

Case 1. $\rho=\sigma=1$. Then the critical relations reduce to

$$
\begin{aligned}
\mathbf{1}+L(z) \tau & =\iota(\mathbf{1}+L(y) \tau) \iota^{-1} L(v) \\
& =L(v)+L\left({ }^{\iota} y^{\tau} v\right) \tau
\end{aligned}
$$

By (2.2.1) this is equivalent to $v=1, z={ }^{\iota} y$, and we are done.

Case 2. $\rho=\sigma=\tau$.

Here the critical relations reduce to ${ }^{\tau} u^{\tau} v v^{-1}=1$, i.e., $u={ }^{\tau} v v^{-1}$, and

$$
\begin{aligned}
\mathbf{1}+L(z) \tau & =L\left({ }^{\tau} v^{-1} v\right) \iota(\mathbf{1}+L(y) \tau) \iota{ }^{-1} L\left({ }^{\tau} v\right) \\
& =L(v)+L\left({ }^{\tau} v^{-1} v^{2 \iota} y\right) \tau
\end{aligned}
$$

which again is equivalent to $v=1, z={ }^{\iota} y$.

e) Here we may also assume $\rho=\sigma$, and also distinguish the following cases.

Case 1. $\rho=\sigma=\mathbf{1}$.

This time the critical relations attain the form

$$
\begin{aligned}
L(c)+L(z) \tau & =\iota(L(c)+L(y) \tau) \iota{ }^{-1} L(v) \\
& =L\left({ }^{\iota} c v\right)+L\left({ }^{\iota} y \tau\right) \tau
\end{aligned}
$$

which is equivalent to

$$
c={ }^{\iota} c v, z={ }^{\iota} y^{\tau} v \text {. }
$$

By $2.7 \mathrm{~b}$ ) this implies $\iota=\mathbf{1}, c v=c, z=y^{\tau} v$, hence $c=c z=c y^{\tau} v=c^{\tau} v$. Therefore $\left.2.7 \mathrm{c}\right)$ yields $v=1, z=y$.

Case 2. $\rho=\sigma=\tau$.

We have to deal with the critical relations ${ }^{\tau} u^{\tau} v v^{-1}=1$, i.e., $u={ }^{\tau} v v^{-1}$, and

$$
\begin{aligned}
L(c)+L(z) \tau & =L\left({ }^{\tau} v^{-1} v\right) \iota(L(c)+L(y) \tau) \iota{ }^{-1} L\left({ }^{\tau} v\right) \\
& =L\left({ }^{\iota} c v\right)+L\left({ }^{\tau} v^{-1} v^{2 \iota} y\right),
\end{aligned}
$$

which is equivalent to

$$
c={ }^{\iota} c v, z={ }^{\tau} v^{-1} v^{2 \iota} y .
$$

As before, this forces $\iota=\mathbf{1}, c=c v, z={ }^{\tau} v^{-1} v^{2} y$, whence $c^{\tau} v=c^{\tau} v z=c v^{2} y=c$. Again we conclude from this $v=1, z=y$.

f) The critical relations read

$$
c={ }^{\tau} v v^{-1} \iota, h=\iota g \iota^{-1} L(v),
$$

forcing $\iota=1$ by $2.7 \mathrm{~b}$ ) and $c v=c^{\tau} v$. Hence $2.7 \mathrm{c}$ ) gives $v=a 1, h=a g$ for some $a \in k^{\times}$. The converse follows from 1.14.

g) Finally, we have to deal with the critical relations $h=L\left(u^{-1}\right) \iota g \iota^{-1} L(u v)$ and

$$
c=u^{\iota} c={ }^{\iota} c,{ }^{\tau} u^{\tau} v v^{-1}=1,
$$


forcing $\iota=\mathbf{1}, u={ }^{\tau} v v^{-1}, v c={ }^{\tau} v c$, which, again by $2.7 \mathrm{c}$ ), implies $v=a 1, u=1, h=a g$ for some $a \in k^{\times}$.

\section{Regular algebras with nonétale heart}

3.0 In this section, we will be concerned with the problem of classifying regular two-dimensional algebras whose unital heart is not étale. Our solution to this problem strongly depends on whether the unital heart is the algebra of dual numbers $(1.7 \mathrm{~b})$ ) or an inseparable quadratic field extension of characteristic two $(1.7 \mathrm{c})$ ). To a considerable extent, however, we will be able to treat both cases simultaneously.

3.1 A common language. Let $K$ be unital two-dimensional $k$-algebra which is not étale. Then there exists an element $\theta \in K$ satisfying $K=k[\theta]$ and $\theta^{2}=\mu 1$ for some $\mu \in k$. Indeed, if $K=k[\varepsilon], \varepsilon^{2}=0$, is the algebra of dual numbers, we may put $\theta=\varepsilon$ and then have $\mu=0$; on the other hand, if $K / k$ is an inseparable quadratic field extension of characteristic two, any $\theta \in K-k 1$ will do, forcing $\mu$ to be nonzero.

This being so, the subgroup

$$
\Gamma=\left\{t \in k^{\times} \mid t^{2} \mu=\mu\right\}
$$

of $k^{\times}$, which agrees with all of $k^{\times}$or $\{1\}$ according as $\mu$ is zero or not, acts effectively on $K$ by automorphisms via

$$
(t, a 1+b \theta) \longmapsto{ }^{t}(a 1+b \theta)=a 1+t b \theta
$$

for $t \in \Gamma, a, b \in k$. In fact, every ( $k$-linear) automorphism of $K$ has the form

$$
\sigma_{t}: K \longrightarrow K, x \longmapsto{ }^{t} x
$$

for some $t \in \Gamma$.

It is easy to see that the decomposition (2.2.1), which was so crucial to the classification of algebras with étale heart, always breaks down in the nonétale case. The following result serves as an adequate substitute for this deficiency.

3.2 Proposition. Let $K \in \operatorname{Alg}(V)$ be unital but not étale. Then, notations being as in 3.1, the $k$-linear map $\partial: V \rightarrow V$ given by $\partial(1)=0, \partial(\theta)=1$ is a $\tau$-derivation of $K$, so

$$
\partial(x y)=\partial(x) y+{ }^{\tau} x \partial(y) \quad(x, y \in V)
$$

or, equivalently,

$$
\partial L(x)=L(\partial(x))+L\left({ }^{\tau} x\right) \partial \quad(x \in V) .
$$

Furthermore, we have the decomposition

$$
\operatorname{End}_{k}(V)=L(K) \oplus L(K) \partial
$$

as a direct sum of subspaces and

$$
\operatorname{det}(L(x)+L(y) \partial)=n(x)+\Delta(x \wedge y) \quad(x, y \in V)
$$


where $\Delta$ stands for the $k$-linear isomorphism $\bigwedge^{2} V \stackrel{\sim}{\longrightarrow} k$ sending $1 \wedge \theta$ to 1. Finally, the relation

$$
\sigma_{t} \partial \sigma_{t}^{-1}=t^{-1} \partial
$$

holds for all $t \in \Gamma$ and

$$
\tau^{\tau}(a 1+b \theta)=a 1-b \theta, n(a 1+b \theta)=a^{2}-\mu b^{2}
$$

for all $a, b \in k$.

Proof. The first part of (6), (1) (or (2)) and (5) are checked by direct verification. In order to establish (3), it suffices to show that the equation $L(x)+L(y) \partial=0(x, y \in V)$ has only the trivial solution $x=y=0$, which follows by applying the left-hand side first to 1 and then to $\theta$. Identifying $\operatorname{End}_{k}(V)=\operatorname{Mat}_{2}(k)$ relative to the basis $(1, \theta)$ of $V$ over $k$, we conclude

$$
L(x)=\left(\begin{array}{cc}
a & \mu b \\
b & a
\end{array}\right), \partial=\left(\begin{array}{ll}
0 & 1 \\
0 & 0
\end{array}\right)
$$

for $x=a 1+b \theta \in V, a, b \in k$. Hence the second part of (6) is clear and (4) follows by explicit computation.

3.3 The $\tau$-derivation $\partial$ of $K$ defined in 3.2 is not intrinsic but depends on the generator $\theta$ of $K$ over $k$. More precisely, choosing another generator subject to the conditions spelled out in 3.1 amounts to replacing $\partial$ by $t \partial$ for some $t \in k^{\times}$.

In contrast to the étale case (cf. 2.3, 2.12), tight enumeration and classification of regular algebras with nonétale heart can be phrased all at once in a single theorem, strongly dependent, however, on the precise nature of the unital heart. As before, all algebras considered are tacitly assumed to be contained in $\operatorname{Alg}(V)$.

3.4 Classification Theorem for Algebras with Dual Heart. Let $K=k[\varepsilon], \varepsilon^{2}=0$, be the algebra of dual numbers and $A$ a regular $k$-algebra of dimension two with unital heart $K$. Then $A$ is isomorphic to precisely one of the following.
a) $K$.
b) $K^{(1, L(b \varepsilon)+\partial)}$ where $b \in k^{\times}$is unique $\bmod k^{\times 2}$.
c) $K^{(\mathbf{1}, L(1+b \varepsilon)+\partial)}$ where $b \in k-\{1\}$ is unique.
d) $K^{(\mathbf{1}, a \mathbf{1}+L(\varepsilon) \partial)}$ where $a \in k-\{0,-1\}$ is unique.
e) $K^{(L(b \varepsilon)+\partial, g)}, b \in k^{\times}, g \in \mathrm{GL}(V)$.
f) $K^{(a \mathbf{1}+L(\varepsilon) \partial, L(b \varepsilon)+\partial)}$ where $a \in k-\{0,1,-1\}$ is unique and $b \in k^{\times}$is unique $\bmod k^{\times 2}$.
g) $K^{(a \mathbf{1}+L(\varepsilon) \partial, L(1+b \varepsilon)+\partial)}$ where $a \in k-\{0,1,-1\}$ and $b \in k-\{1\}$ are unique.
h) $K^{(\mathbf{1}+L(\varepsilon) \partial, L(b \varepsilon)+\partial)}$ where char $k \neq 2$ and $b \in k^{\times}$is unique $\bmod k^{\times 2}$.
i) $K^{(\mathbf{1}+L(\varepsilon) \partial, L(b \varepsilon)+L(1+\varepsilon) \partial)}$ where char $k \neq 2$ and $b \in k^{\times}$is unique.
j) $K^{(a \mathbf{1}+L(\varepsilon) \partial, \alpha \mathbf{1}+L(\varepsilon) \partial)}$ where $a, \alpha \in k-\{0,-1\}$ are unique. 
k) $K^{(a 1+L(\varepsilon) \partial, L(\alpha 1+\varepsilon)+L(\varepsilon) \partial)}$ where $a \in k-\{0,-1\}$ is unique and $\alpha=-\frac{a}{1+a}$.

l) $K^{(a \mathbf{1}+L(\varepsilon) \partial, \mathbf{1})}$ where $a \in k-\{0,-1\}$ is unique.

Furthermore, given $b, b^{\prime} \in k^{\times}$and $g, g^{\prime} \in \mathrm{GL}(V)$, we have

$$
K^{(L(b \varepsilon)+\partial, g)} \cong K^{\left(L\left(b^{\prime} \varepsilon\right)+\partial, g^{\prime}\right)}
$$

if and only if there are elements $t, a \in k^{\times}$satisfying $b^{\prime}=b t^{2}$ and $g^{\prime}=a \sigma_{t} g \sigma_{t}^{-1}$.

Remark. In $3.4 \mathrm{~b}$ ), uniqueness $\bmod k^{\times 2}$ of $b \in k^{\times}$is supposed to mean that elements $b, b^{\prime} \in k^{\times}$give rise to isomorphic algebras if and only if $b \equiv b^{\prime} \bmod k^{\times 2}$. Analogous interpretations prevail in the other cases of the classification list, as in $3.6 \mathrm{~b}$ ), 4.11 below.

3.5 In order to phrase the analogue of 3.4 for an iseparable quadratic field extension $K / k$ of characteristic two rather than the algebra of dual numbers, we require the logarithmic derivative, i.e., the group homomorphism

$$
\partial \log : K^{\times} \longrightarrow K, x \longmapsto(\partial \log )(x)=x^{-1} \partial(x),
$$

whose kernel is $k^{\times} 1$ and which plays a rôle similar to the one of the norm in the étale case, see 2.3 a),c), 2.12 a,c) above. Notice, however, that $\partial \log$ is uniquely determined by $K / k$ only up to nonzero scalar factors (3.3).

3.6 Classification Theorem for Algebras with Inseparable Heart. Let $K / k$ be an inseparable quadratic field extension of characteristic two and $A$ a regular twodimensional $k$-algebra with unital heart $K$. Then $A$ is isomorphic to precisely one of the following.

a) $K$.

b) $K^{(1, L(x)+\partial)}$ where $x \in V$ satisfying $n(x)+\Delta(x \wedge 1) \neq 0$ is unique.

c) $K^{(L(x)+\partial, g)}$ where $x \in V$ satisfies $n(x)+\Delta(x \wedge 1) \neq 0$ and $g \in \operatorname{GL}(V)$.

Furthermore, given $x, x^{\prime} \in V$ satisfying $n(x)+\Delta(x \wedge 1) \neq 0 \neq n\left(x^{\prime}\right)+\Delta\left(x^{\prime} \wedge 1\right)$ and $g, g^{\prime} \in \mathrm{GL}(V)$, we have

$$
K^{(L(x)+\partial, g)} \cong K^{\left(L\left(x^{\prime}\right)+\partial, g^{\prime}\right)}
$$

if and only if there exists an element $a \in k^{\times}$such that either $x^{\prime}=x$ and $g^{\prime}=a g$ or

$$
x \neq x^{\prime}, x \equiv x^{\prime} \operatorname{modim} \partial \log \text { and } g^{\prime}=a g L\left(x-x^{\prime}\right) .
$$

3.7 We will try as long as possible to prove these two results simultaneously and therefore return to the general setup described in 3.1. Our first aim is to establish a number of technical results that will turn out to be useful later on. 
3.8 Lemma. Given $x, y \in V, u, v \in K^{\times}, t \in \Gamma$, we have

$$
\begin{aligned}
& L\left(v^{-1}\right) \sigma_{t}(L(x)+L(y) \partial) \sigma_{t}^{-1} L(u v)= \\
& L\left(u^{t} x+t^{-1}\left[\partial(u)+{ }^{\tau} u v^{-1} \partial(v)\right]^{t} y\right)+L\left(t^{-1 \tau} u^{\tau} v v^{-1 t} y\right) \partial .
\end{aligned}
$$

Proof. Expanding the left-hand side yields

$$
\begin{aligned}
& L\left(v^{-1}\right) \sigma_{t}(L(x)+L(y) \partial) \sigma_{t}^{-1} L(u v) \\
& =L\left(v^{-1}\right)\left(L\left({ }^{t} x\right)+L\left({ }^{t} y\right) \sigma_{t} \partial \sigma_{t}^{-1}\right) L(u v) \\
& =L\left(v^{-1}\right)\left(L\left({ }^{t} x\right)+t^{-1} L\left({ }^{t} y\right) \partial\right) L(u v) \\
& =L\left(u^{t} x\right)+t^{-1} L\left(v^{-1} t y\right)\left(L(\partial(u v))+L\left({ }^{\tau} u^{\tau} v\right) \partial\right) \\
& =L\left(u^{t} x+t^{-1}\left[\partial(u)+{ }^{\tau} u v^{-1} \partial(v)\right]^{t} y\right)+L\left(t^{-1 \tau} u^{\tau} v v^{-1 t} y\right) \partial
\end{aligned}
$$

the latter being a consequence of (3.2.1).

3.9 Lemma. For $x, y, x^{\prime}, y^{\prime} \in V$ satisfying $n(x)+\Delta(x \wedge y) \neq 0 \neq n\left(x^{\prime}\right)+\Delta\left(x^{\prime} \wedge y^{\prime}\right)$ and $g, g^{\prime} \in$ $\mathrm{GL}(V)$, the following statements are equivalent.

(i) $K^{(L(x)+L(y) \partial, g)} \cong K^{\left(L\left(x^{\prime}\right)+L\left(y^{\prime}\right) \partial, g^{\prime}\right)}$.

(ii) There are elements $u, v \in K^{\times}, t \in \Gamma$ satisfying

$$
\begin{aligned}
& x^{\prime}=u^{t} x+t^{-1}\left[\partial(u)+{ }^{\tau} u v^{-1} \partial(v)\right]^{t} y, \\
& y^{\prime}=t^{-1 \tau} u^{\tau} v v^{-1 t} y, \\
& g^{\prime}=L\left(u^{-1}\right) \sigma_{t} g \sigma_{t}^{-1} L(u v) .
\end{aligned}
$$

In this case, $y$ is invertible (resp. zero) if and only if $y^{\prime}$ is.

Proof. The first part follows immediately from the isomorphism criterion 1.12 combined with 3.1, (3.2.3) and 3.8. The second part is obvious.

3.10 Lemma. Let $(f, g) \in G$. Then there exists an element $\left(f^{\prime}, g^{\prime}\right) \in G$ such that $K^{(f, g)} \cong K^{\left(f^{\prime}, g^{\prime}\right)}$ and $f^{\prime}$ satisfies one of the following mutually exclusive conditions.

a) $f^{\prime}=1$.

b) $f^{\prime}=L(x)+\partial$ for some $x \in K$ having $n(x)+\Delta(x \wedge 1) \neq 0$.

c) $f^{\prime}=L(x)+L(\varepsilon) \partial$, where $K$ is the algebra of dual numbers and $x \in K$ has $n(x)+\Delta(x \wedge \varepsilon) \neq 0$.

Proof. (3.2.3), (3.2.4) allow us to write $f=L(x)+L(y) \partial$ where $x, y \in K$ satisfy $n(x)+\Delta(x \wedge y) \neq 0$. Hence, if $y=0, x$ is invertible and putting $v=t=1, u=x^{-1}$ in 3.9 leads to a). If $y$ is invertible, we put $v=t=1, u={ }^{\tau} y^{-1}$ in 3.9 and arrive at b). Finally, if $y$ is neither invertible nor zero, $K$ is the algebra of dual numbers and $y=b \varepsilon$ for some $b \in k^{\times}$, so putting $v=t=1, u=b^{-1} 1$ in 3.9 leads to c). Disjointness of cases a), b), c) follows from the final statement of 3.9. 
3.11 We proceed by discussing the cases a) - c) of 3.10 separately. Starting with a), we will be concerned with the isomorphism problem for algebras of the form $K^{(\mathbf{1}, g)}, g \in \mathrm{GL}(V)$. Note first that the specialization $v=1, u=v$ in 3.8 gives

$$
\begin{aligned}
& \sigma_{t}(L(x)+L(y) \partial) \sigma_{t}^{-1} L(v)= \\
& L\left(v^{t} x+t^{-1} \partial(v)^{t} y\right)+L\left(t^{-1} v^{t} y\right) \partial
\end{aligned}
$$

for all $t \in \Gamma, v \in K^{\times}, x, y \in V$. Furthermore, by 3.9 with $x=x^{\prime}=1, y=y^{\prime}=0$, the algebras $K^{(\mathbf{1}, g)}$ and $\left.K^{\left(\mathbf{1}, g^{\prime}\right)}, g, g^{\prime} \in \mathrm{GL}(V)\right)$ are isomorphic if and only if there are elements $u, v \in K^{\times}, t \in \Gamma$ satisfying $u=1$ and $g^{\prime}=\sigma_{t} g \sigma_{t}^{-1} L(v)$. Hence we obtain

3.12 Lemma. For all $g, g^{\prime} \in \mathrm{GL}(V)$, the algebras $K^{(\mathbf{1}, g)}$ and $K^{\left(\mathbf{1}, g^{\prime}\right)}$ are isomorphic if and only if there are elements $t \in \Gamma, v \in K^{\times}$such that $g^{\prime}=\sigma_{t} g \sigma_{t}^{-1} L(v)$.

3.13 Lemma. For every $g \in \mathrm{GL}(V)$, precisely one of the following holds.

a) $K^{(\mathbf{1}, g)} \cong K$.

b) $K^{(\mathbf{1}, g)} \cong K^{(\mathbf{1}, L(x)+\partial)}$, where $x \in V$ satisfies $n(x)+\Delta(x \wedge 1) \neq 0$.

c) $K^{(\mathbf{1}, g)} \cong K^{(\mathbf{1}, L(x)+L(\varepsilon) \partial)}$, where $K$ is the algebra of dual numbers and $x \in K$ satisfies $n(x)+$ $\Delta(x \wedge \varepsilon) \neq 0$.

Proof. We argue as in the proof of 3.10, using (3.11.1) and 3.12 rather than 3.9. First write $g=L(x)+L(y) \partial$ where $x, y \in V$ satisfy $n(x)+\Delta(x \wedge y) \neq 0((3.2 .3),(3.2 .4))$. If $y=0$, then $x$ is invertible, allowing us to put $t=1, v=x^{-1}$ in 3.12. By (3.11.1), we arrive at a). If $y$ is invertible, we put $t=1, v={ }^{\tau} y^{-1}$ in 3.12 and end up with b). Finally, if $y$ is neither invertible nor zero, $K$ is the algebra of dual numbers and $y=b \varepsilon$ for some $b \in k^{\times}$, so 3.12 with $t=1, v=b^{-1} 1$ gives c).

3.14 Lemma. If $x, x^{\prime} \in V$ satisfy $n(x)+\Delta(x \wedge 1) \neq 0 \neq n\left(x^{\prime}\right)+\Delta\left(x^{\prime} \wedge 1\right)$, the following statements are equivalent.

(i) $K^{(\mathbf{1}, L(x)+\partial)} \cong K^{\left(\mathbf{1}, L\left(x^{\prime}\right)+\partial\right)}$.

(ii) There exists an element $t \in \Gamma$ such that $x^{\prime}=t^{t} x$.

Proof. (i) holds iff there are elements $t \in \Gamma, v \in K^{\times}$satisfying

$$
\begin{aligned}
L\left(x^{\prime}\right)+\partial & =\sigma_{t}(L(x)+\partial) \sigma_{t}^{-1} L(v) \\
& =L\left(v^{t} x+t^{-1} \partial(v)\right)+L\left(t^{-1 \tau} v\right) \partial
\end{aligned}
$$

which amounts to $v=t 1$ and $x^{\prime}=t^{t} x$.

3.15 Lemma. Suppose $K$ is the algebra of dual numbers and $x, x^{\prime} \in V$ satisfy $n(x)+\Delta(x \wedge \varepsilon) \neq$ $0 \neq n\left(x^{\prime}\right)+\Delta\left(x^{\prime} \wedge \varepsilon\right)$. Then the following statements are equivalent.

(i) $K^{(\mathbf{1}, L(x)+L(\varepsilon) \partial)} \cong K^{\left(\mathbf{1}, L\left(x^{\prime}\right)+L(\varepsilon) \partial\right)}$. 
(ii) There are elements $t \in k^{\times}, \beta \in k$ such that

$$
x^{\prime}=(1+\beta \varepsilon)^{t} x+\beta \varepsilon .
$$

Proof. Arguing as in the proof of 3.14, we see that (i) holds iff there are elements $t \in \Gamma, v \in K^{\times}$ satisfying

$$
x^{\prime}=v^{t} x+\partial(v) \varepsilon, \varepsilon={ }^{\tau} v \varepsilon .
$$

Writing $v=\alpha 1+\beta \varepsilon$ with $\alpha, \beta \in k$, the second one of these equations amounts to $\alpha=1$. Hence the assertion follows.

3.16 Lemma. Suppose $x, x^{\prime} \in V$ satisfy $n(x)+\Delta(x \wedge 1) \neq 0 \neq n\left(x^{\prime}\right)+\Delta\left(x^{\prime} \wedge 1\right)$ and let $g, g^{\prime} \in$ $\mathrm{GL}(V)$. Then the following statements are equivalent.

(i) $K^{(L(x)+\partial, g)} \cong K^{\left(L\left(x^{\prime}\right)+\partial, g^{\prime}\right)}$.

(ii) There are elements $t \in \Gamma, v \in K^{\times}$such that

$$
x^{\prime}=t^{\tau} v v^{-1 t} x-v^{-1} \partial(v), g^{\prime}=L\left({ }^{\tau} v^{-1} v\right) \sigma_{t} g \sigma_{t}^{-1} L\left({ }^{\tau} v\right) .
$$

Proof. By 3.9, (i) holds iff there are elements $u, v \in K^{\times}, t \in \Gamma$ satisfying

$$
\begin{aligned}
x^{\prime} & =u^{t} x+t^{-1}\left[\partial(u)+{ }^{\tau} u v^{-1} \partial(v)\right], \\
1 & =t^{-1} u^{\tau} v v^{-1} \\
g^{\prime} & =L\left(u^{-1}\right) \sigma_{t} g \sigma_{t}^{-1} L(u v) .
\end{aligned}
$$

Here (2) amounts to

$$
u=t^{\tau} v v^{-1} \text {. }
$$

Since

$$
\partial\left({ }^{\tau} v\right)=-\partial(v)
$$

we conclude that (1) and (2) are equivalent to (4) and

$$
\begin{aligned}
x^{\prime}= & t^{\tau} v v^{-1 t} x+\partial\left({ }^{\tau} v v^{-1}\right)+{ }^{\tau} v^{-1} \partial(v) \\
= & t^{\tau} v v^{-1 t} x-\partial(v) v^{-1}+v \partial\left(v^{-1}\right) \\
& +{ }^{\tau} v^{-1} \partial(v) \\
= & t^{\tau} v v^{-1 t} x-v^{-1} \partial(v)+n(v)^{-1} v \partial\left({ }^{\tau} v\right) \\
& +n\left(v^{-1}\right) v \partial(v) \\
= & t^{\tau} v v^{-1 t} x-v^{-1} \partial(v)
\end{aligned}
$$

Substituting (4) into (3) completes the proof. 
3.17 Lemma. Let $K / k$ be an inseparable quadratic field extension of characteristic two and $x \in$ $K$. Then the following statements are equivalent.

(i) $x \in \operatorname{im} \partial \log$.

(ii) $\partial(x)=x^{2}$.

(iii) If $x \in K^{\times}$then $x=(\partial \log )(x)$.

Proof. The implications (ii) $\Rightarrow$ (iii) $\Rightarrow$ (i) are obvious. In order to establish (i) $\Rightarrow$ (ii), suppose $x=(\partial \log )(v)$ for some $v \in K^{\times}$. Then

$$
\begin{aligned}
\partial(x) & =\partial\left(v^{-1} \partial(v)\right)=\partial\left(v^{-1}\right) \partial(v)+v^{-1} \partial^{2}(v) \\
& =-v^{-2} \partial(v)^{2} \\
& =\left(v^{-1} \partial(v)\right)^{2}=x^{2} .
\end{aligned}
$$

(since $\left.\partial^{2}=0\right)$

3.18 We are now prepared to prove 3.6 and first note that the classes a), b), c) are disjoint by the final statement of 3.9 combined with (1.2.2). Next let $A \in \operatorname{Alg}(V)$ be regular with unital heart $K$. Then $A \cong K^{(f, g)}$ for some $f, g \in \mathrm{GL}(V)$, and by 3.10 it suffices to consider the following cases.

Case 1. $f=\mathbf{1}$.

By 3.13, $A$ satisfies either a) or b) of 3.6, and $x$ in case b) is unique since $\Gamma=\{1\}(3.14)$.

Case 2. $f=L(x)+\partial, x \in V, n(x)+\Delta(x \wedge 1) \neq 0$.

Then $A$ satisfies $3.6 \mathrm{c}$ ) whence it suffices to establish the final statement of the theorem. To this end, suppose $x, x^{\prime} \in V$ satisfy $n(x)+\Delta(x \wedge 1) \neq 0 \neq n\left(x^{\prime}\right)+\Delta\left(x^{\prime} \wedge 1\right)$. By $3.16, K^{(L(x)+\partial, g)} \cong K^{\left(L\left(x^{\prime}\right)+\partial, g^{\prime}\right)}$ iff there exists an element $v \in K^{\times}$satisfying

$$
x^{\prime}=x+(\partial \log )(v), \quad g^{\prime}=g L(v) .
$$

For $x^{\prime}=x$, this is equivalent to $v=a 1$ and $g^{\prime}=a g$ for some $a \in k^{\times}$. For $x^{\prime} \neq x,(1)$ combines with 3.17 to yield $x-x^{\prime}=(\partial \log )\left(x-x^{\prime}\right)$, hence $v=a\left(x-x^{\prime}\right)$ and $g^{\prime}=a g L\left(x-x^{\prime}\right)$ for some $a \in k^{\times}$. This completes the proof of the Classification Theorem 3.6.

For the rest of this section we therefore assume that $K$ is the algebra of dual numbers, forcing $\mu=0$ and $\Gamma=k^{\times}$in 3.1 .

3.19 Lemma. Suppose $x, x^{\prime} \in V$ satisfy $n(x)+\Delta(x \wedge \varepsilon) \neq 0 \neq n\left(x^{\prime}\right)+\Delta\left(x^{\prime} \wedge \varepsilon\right)$ and let $g, g^{\prime} \in$ $\mathrm{GL}(V)$. Then the following statements are equivalent.

(i) $K^{(L(x)+L(\varepsilon) \partial, g)} \cong K^{\left(L\left(x^{\prime}\right)+L(\varepsilon) \partial, g^{\prime}\right)}$.

(ii) There are elements $t \in k^{\times}, u, v \in K^{\times}$such that

$$
\begin{aligned}
x^{\prime} & =u^{t} x+\left[\partial(u)+{ }^{\tau} u v^{-1} \partial(v)\right] \varepsilon, \\
\varepsilon & ={ }^{\tau} u^{\tau} v v^{-1} \varepsilon \\
g^{\prime} & =L\left(u^{-1}\right) \sigma_{t} g \sigma_{t}^{-1} L(u v) .
\end{aligned}
$$

In this case, $x$ is invertible if and only if $x^{\prime}$ is.

Proof. The first part is simply a restatement of 3.9 with $y=y^{\prime}=\varepsilon$. The second part is clear. 
3.20 We record a few explicit formulae whose routine verification is left to the reader. Let $a, b, \alpha, \beta, \delta \in k, \gamma, t \in k^{\times}$and put

$$
x=a 1+b \varepsilon, u=\alpha 1+\beta \varepsilon, v=\gamma 1+\delta \varepsilon .
$$

Then

$$
\begin{aligned}
t^{\tau} v v^{-1 t} x-v^{-1} \partial(v) & =\left(t a-\gamma^{-1} \delta\right) 1+\left(t^{2}\left(b-a^{2}\right)+\left(t a-\gamma^{-1} \delta\right)^{2}\right) \varepsilon \\
{ }^{\tau} u^{\tau} v v^{-1} & =\alpha 1-\left(\beta+2 \alpha \gamma^{-1} \delta\right) \varepsilon \\
\partial(u)+{ }^{\tau} u v^{-1} \partial(v) & =\left(\beta+\alpha \gamma^{-1} \delta\right) 1-\gamma^{-2} \delta(\alpha \delta+\beta \gamma) \varepsilon \\
{ }^{\tau} u^{\tau} v v^{-1} \varepsilon & =\alpha \varepsilon \\
u^{t} x+\left[\partial(u)+{ }^{\tau} u v^{-1} \partial(v)\right] \varepsilon & =\alpha a 1+\left(\beta(a+1)+\alpha\left(t b+\gamma^{-1} \delta\right)\right) \varepsilon .
\end{aligned}
$$

3.21 Lemma. For $a, a^{\prime} \in k-\{0,-1\}$ and $g, g^{\prime} \in \mathrm{GL}(V)$, the following statements are equivalent.

(i) $K^{(a \mathbf{1}+L(\varepsilon) \partial, g)} \cong K^{\left(a^{\prime} \mathbf{1}+L(\varepsilon) \partial, g^{\prime}\right)}$.

(ii) $a=a^{\prime}$, and there are $\beta \in k, \gamma, t \in k^{\times}$such that

$$
g^{\prime}=L\left(u^{-1}\right) \sigma_{t} g \sigma_{t}^{-1} L(u v)
$$

where

$$
u=1+\beta \varepsilon, v=\gamma(1-\beta(a+1)) \varepsilon
$$

Proof. We put $x=a 1, x^{\prime}=a^{\prime} 1$. Then (i) holds iff there are elements $t \in k^{\times}, u, v \in K^{\times}$satisfying the conditions of 3.19 (ii). By (3.20.4), (3.20.5) these conditions are fulfilled iff $u=1+\beta \varepsilon, v=$ $\gamma 1+\delta \varepsilon\left(\beta, \delta \in k, \gamma \in k^{\times}\right), a=a^{\prime}, \beta(a+1)+\gamma^{-1} \delta=0$ and $g^{\prime}$ is as indicated. The assertion follows.

3.22 Lemma. Let $a \in k-\{0,-1\}$ and suppose $x, y, x^{\prime}, y^{\prime} \in V$ satisfy $n(x)+\Delta(x \wedge y) \neq 0 \neq$ $n\left(x^{\prime}\right)+\Delta\left(x^{\prime} \wedge y^{\prime}\right)$. Then the following statements are equivalent.

(i) $K^{(a \mathbf{1}+L(\varepsilon) \partial, L(x)+L(y) \partial)} \cong K^{\left(a \mathbf{1}+L(\varepsilon) \partial, L\left(x^{\prime}\right)+L\left(y^{\prime}\right) \partial\right)}$.

(ii) There are $\beta \in k, \gamma, t \in k^{\times}$such that

$$
u=1+\beta \varepsilon, v=\gamma(1-\beta(a+1) \varepsilon)
$$

satisfy

$$
\begin{aligned}
x^{\prime} & =v^{t} x+t^{-1}\left[\partial(v)+{ }^{\tau} v u^{-1} \partial(u)\right]^{t} y, \\
y^{\prime} & =t^{-1 \tau} v^{\tau} u u^{-1 t} y .
\end{aligned}
$$

In this case, $y$ is invertible (resp. 0) if and only if $y^{\prime}$ is.

Proof. By 3.21, (i) holds iff there are $\beta \in k, \gamma, t \in k^{\times}$such that $u, v$ as indicated satisfy

$$
L\left(x^{\prime}\right)+L\left(y^{\prime}\right) \partial=L\left(u^{-1}\right) \sigma_{t}(L(x)+L(y) \partial) \sigma_{t}^{-1} L(u v) .
$$

By 3.8 with $u, v$ interchanged, this is equivalent to the relations for $x^{\prime}, y^{\prime}$ stated in (ii). The rest is obvious. 
3.23 Given $\alpha, \beta \in k, \gamma, t \in k^{\times}$and setting

$$
u=1+\beta \varepsilon, v=\gamma(1-\beta(a+1) \varepsilon)
$$

as in 3.22 , it is straightforward to check, using (3.20.2), (3.20.3) with $u, v$ interchanged, that

$$
\begin{aligned}
& { }^{\tau} v^{\tau} u u^{-1}=\gamma(1+\beta(a-1) \varepsilon), \\
& \partial(v)+{ }^{\tau} v u^{-1} \partial(u)=-\beta \gamma a(1+\beta \varepsilon) .
\end{aligned}
$$

3.24 We have finally reached the point where we are able to tackle the Classification Theorem 3.4. Let $A$ be a twodimensional regular algebra with unital heart $K$, so $A \cong K^{(f, g)}$ for some $(f, g) \in G$. By 3.10 , we may assume that $f$ belongs to one of the following mutually exclusive cases.

Case $1 . f=\mathbf{1}$.

Then we will show that $A$ is isomorphic to one of the algebras in 3.4 a) - d). To begin with, 3.13 up to isomorphism leaves us with the following disjoint subcases.

Case 1.1. $A \cong K$.

Then we are in the situation of $3.4 \mathrm{a}$ ).

Case 1.2. $g=L(x)+\partial$ where $x \in V$ satisfies $n(x)+\Delta(x \wedge 1) \neq 0$.

Writing $x=a 1+b \varepsilon$ with $a, b \in k$, we therefore have $a^{2} \neq b$. Also, by 3.14 , we are allowed to replace $x$ by

$$
t^{t} x=t(a 1+t b \varepsilon)=t a 1+t^{2} b \varepsilon
$$

for any $t \in k^{\times}$. Then either $a=0$, which yields case $3.4 \mathrm{~b}$ ) and, thanks to (1), makes $b$ indeed unique $\bmod k^{\times 2}$, or $a \neq 0$, and (1) allows us to assume $a=1$, bringing us to case $3.4 \mathrm{c}$ ) and showing that $b \in k-\{1\}$ is unique.

Case 1.3. $g=L(x)+L(\varepsilon) \partial$ where $x \in V$ satisfies $n(x)+\Delta(x \wedge \varepsilon) \neq 0$.

For $x=a 1+b \varepsilon, a, b \in k$, this means $a^{2}+a \neq 0$, i.e., $a \neq 0,-1$. Here 3.15 allows us to replace $x$ by

$$
(1+\beta \varepsilon)^{t} x+\beta \varepsilon=a 1+(t b+\beta(a+1)) \varepsilon
$$

for any $\beta \in k, t \in k^{\times}$. For $t=1, \beta=-b(a+1)^{-1}$, this brings us to case $\left.3.4 \mathrm{~d}\right)$, where $a$ is unique.

Case 2. $f=L(x)+\partial$ where $x \in V$ satisfies $n(x)+\Delta(x \wedge 1) \neq 0$.

This means $x=a 1+b \varepsilon$ where $a, b \in k$ satisfy $a^{2} \neq b$. We will show that $A$ belongs to case $\left.3.4 \mathrm{e}\right)$. Consulting 3.16 and (3.20.1), we may replace $x$ by

$$
\left(t a-\gamma^{-1} \delta\right) 1+\left(t^{2}\left(b-a^{2}\right)+\left(t a-\gamma^{-1} \delta\right)^{2}\right) 1
$$

for any $t, \gamma \in k^{\times}, \delta \in k$. By a proper choice of these parameters, we may assume $a=0$, so we are in case $3.4 \mathrm{e})$. Furthermore, given $b, b^{\prime} \in k^{\times}$and $g, g^{\prime} \in \mathrm{GL}(V)$, the algebras $K^{(L(b \varepsilon)+\partial, g)}$ and $K^{\left(L\left(b^{\prime} \varepsilon\right)+\partial, g^{\prime}\right)}$ are isomorphic by 3.16, (3.20.1) and (2) combined iff there are $t, \gamma \in k^{\times}$satisfying $b^{\prime}=t^{2} b$ and $g^{\prime}=\gamma \sigma_{t} g \sigma_{t}^{-1}$. This settles the final statement of 3.4 as well.

Case 3. $f=L(x)+L(\varepsilon) \partial$ where $x \in V$ satisfies $n(x)+\Delta(x \wedge \varepsilon) \neq 0$.

For $x=a 1+b \varepsilon, a, b \in k$, this means $a \neq 0,-1$. We will show that $A$ belongs to one of the case 3.4 f) - 1). Combining 3.19 with (3.20.4), (3.20.5) we are allowed to replace $x$ by

$$
a 1+\left(\beta(a+1)+t b+\gamma^{-1} \delta\right) \varepsilon
$$


for any $\beta, \delta \in k, t, \gamma \in k^{\times}$. Hence we may assume $b=0$, which yields

$$
f=a \mathbf{1}+L(\varepsilon) \partial, a \in k-\{0,-1\} .
$$

Morever, $a$ is uniqely determined by $A$ (3.21). We now use (3.2.3), (3.2.4) to decompose $g$ as

$$
g=L(x)+L(y) \partial,
$$

where $x, y \in V$ satisfy $n(x)+\Delta(x \wedge y) \neq 0$. We write $x=\xi_{1} 1+\xi_{2} \varepsilon\left(\xi_{1}, \xi_{2} \in k\right)$ and consider an additional element $x^{\prime}=\xi_{1}^{\prime} 1+\xi_{2}^{\prime} \varepsilon \in V\left(\xi_{1}^{\prime}, \xi_{2}^{\prime} \in k\right)$. Then we proceed by discussing the subcases $y \in K^{\times}, 0 \neq y \in K-K^{\times}$and $y=0$ separately, which are disjoint by 3.22 .

Case 3.1. $y \in K^{\times}$.

Since we are allowed to multiply $g$ by nonzero scalars (1.14), there is no harm in assuming

$$
y=1+s \varepsilon, s \in k .
$$

Given $\beta \in k, \gamma, t \in k^{\times}$, we apply 3.22 to replace $y$ by

$$
\begin{aligned}
y^{\prime} & =t^{-1 \tau} v^{\tau} u u^{-1 t} y \\
& =t^{-1} \gamma(1+\beta(a-1) \varepsilon)(1+t s \varepsilon) \\
& =t^{-1} \gamma(1+(t s+\beta(a-1)) \varepsilon),
\end{aligned}
$$

so $y^{\prime}$ has the form

$$
y^{\prime}=1+s^{\prime} \varepsilon, s^{\prime} \in k,
$$

if and only if

$$
\gamma=t, s^{\prime}=t s+\beta(a-1)
$$

Case 3.1.1. $a \neq 1$.

Setting $\gamma=t=1, \beta=-(a-1)^{-1} s$, we conclude $y^{\prime}=1$ from (6), (7), allowing us to assume $y=1$. This implies $\xi_{1}^{2}-\xi_{2} \neq 0$. Now suppose $x^{\prime}$ satisfies $\xi_{1}^{\prime 2}-\xi_{2}^{\prime} \neq 0$ as well and put $g^{\prime}=L\left(x^{\prime}\right)+\partial$. Then $A \cong K^{(f, g)} \cong K^{\left(f, g^{\prime}\right)}$ iff there are $\beta \in k, \gamma, t \in k^{\times}$such that $u, v$ as in (3.22.1) satisfy (3.22.2), (3.22.3). The latter is equivalent to (7) with $s=s^{\prime}=0$, forcing $\beta=0, u=1, v=t 1$. Hence $K^{(f, g)} \cong K^{\left(f, g^{\prime}\right)}$ iff, for some $t \in k^{\times}$,

$$
\xi_{1}^{\prime} 1+\xi_{2}^{\prime} \varepsilon=t^{t} x=t \xi_{1} 1+t^{2} \xi_{2} \varepsilon
$$

We are therefore left with the following sub-subcases, which are evidently disjoint.

Case 3.1.1.1. $\xi_{1}=0$.

Then $A$ is as in $3.4 \mathrm{f}$ ) and, by (8), $\xi_{2} \in k^{\times}$is unique $\bmod k^{\times 2}$.

Case 3.1.1.2. $\xi_{1} \neq 0$.

By (8), we may assume $\xi_{1}=1$, so $A$ is as in $3.4 \mathrm{~g}$ ). Conversely, $\xi_{1}=\xi_{1}^{\prime}=1$ in (8) implies $t=1$, $\xi_{2}=\xi_{2}^{\prime}$, forcing $b$ as in $3.4 \mathrm{~g}$ ) to be unique.

Case 3.1.2. $a=1$.

Then char $k \neq 2$ by (3), and (7) reduces to

$$
\gamma=t, s^{\prime}=t s
$$


Again we are left with two disjoint sub-subcases.

Case 3.1.2.1. $s=0$.

Then $y=1=y^{\prime}$ and (9) reduces to $\gamma=t$. As before, we have $\xi_{1}^{2} \neq \xi_{2}$. Assuming $\xi_{1}^{\prime 2} \neq \xi_{2}^{\prime}$ as well, and setting $g^{\prime}=L\left(x^{\prime}\right)+\partial$, we have $K^{(f, g)} \cong K^{\left(f, g^{\prime}\right)}$ iff there are $\beta \in k, \gamma, t \in k^{\times}$such that $u, v$ as in (3.22.1) satisfy $\gamma=t$ and

$$
\begin{aligned}
\xi_{1}^{\prime} 1+\xi_{2}^{\prime} \varepsilon & =x^{\prime} \\
& =v^{t} x+t^{-1}\left[\partial(v)+{ }^{\tau} v u^{-1} \partial(u)\right] \\
& =t(1-2 \beta \varepsilon)\left(\xi_{1}+t \xi_{2} \varepsilon\right)-\beta(1+\beta \varepsilon)
\end{aligned}
$$

i.e.,

$$
\xi_{1}^{\prime}=t \xi_{1}-\beta, \xi_{2}^{\prime}=t\left(t \xi_{2}-2 \beta \xi_{1}\right)-\beta^{2} .
$$

We may therefore assume $\xi_{1}=0$, so $A$ is as in $3.4 \mathrm{~h}$ ). Conversely, if $\xi_{1}=\xi_{1}^{\prime}=0,(10)$ reduces to $\beta=0, \xi_{2}^{\prime}=t^{2} \xi_{2}$. Hence $b$ as in $3.4 \mathrm{~h}$ ) is unique $\bmod k^{\times 2}$.

Case 3.1.2.2. $s \neq 0$.

Then (9) allows us to assume $s=1$, forcing $y=1+\varepsilon$ and $\xi_{1}\left(1+\xi_{1}\right) \neq \xi_{2}$. Assuming $\xi_{1}^{\prime}\left(1+\xi_{1}^{\prime}\right) \neq \xi_{2}^{\prime}$ as well, and setting $g^{\prime}=L\left(x^{\prime}\right)+L(1+\varepsilon) \partial$, we have $K^{(f, g)} \cong K^{\left(f, g^{\prime}\right)}$ iff there are $\beta \in k, \gamma, t \in k^{\times}$such that $u, v$ as in (3.22.1) satisfy (3.22.2), (3.22.3). The latter being equivalent to (9) with $s=s^{\prime}=1$, this amounts to $\gamma=t=1$ and

$$
\begin{aligned}
\xi_{1}^{\prime} 1+\xi_{2}^{\prime} \varepsilon & =x^{\prime} \\
& =v^{t} x+t^{-1}\left[\partial(v)+{ }^{\tau} v u^{-1} \partial(u)\right]^{t} y \\
& =(1-2 \beta \varepsilon)\left(\xi_{1} 1+\xi_{2} \varepsilon\right)-\beta(1+\beta \varepsilon)(1+\varepsilon)
\end{aligned}
$$

i.e.,

$$
\xi_{1}^{\prime}=\xi_{1}-\beta, \xi_{2}^{\prime}=\xi_{2}-\beta\left(2 \xi_{1}+\beta+1\right)
$$

Hence we may assume $\xi_{1}=0$, forcing $A$ to be as in $3.4 \mathrm{i}$ ). Conversely, if $\xi_{1}=\xi_{1}^{\prime}=0$, (11) reduces to $\beta=0, \xi_{2}^{\prime}=\xi_{2}$, and $b$ as in $3.4 \mathrm{i}$ ) is unique.

Case 3.2. $0 \neq y \in K-K^{\times}$.

By 1.14 we may assume $y=\varepsilon$, which implies $\xi_{1} \neq 0,-1$. Assuming $\xi_{1}^{\prime} \neq 0,-1$ as well, and setting $g^{\prime}=L\left(x^{\prime}\right)+L(\varepsilon) \partial$, we have $K^{(f, g)} \cong K^{\left(f, g^{\prime}\right)}$ iff there are $\beta \in k, \gamma, t \in k^{\times}$such that $u, v$ as in (3.22.1) satisfy (3.22.2), (3.22.3). The latter amounts to $\varepsilon={ }^{\tau} v^{\tau} u u^{-1} \varepsilon=\gamma \varepsilon$ (by 3.23.1)), i.e., to $\gamma=1$. Then the former reads

$$
\begin{aligned}
\xi_{1}^{\prime} 1+\xi_{2}^{\prime} \varepsilon= & (1-\beta(a+1) \varepsilon)\left(\xi_{1} 1+t \xi_{2} \varepsilon\right) \\
& -\beta a(1+\beta \varepsilon) \varepsilon
\end{aligned}
$$

and hence is equivalent to

$$
\xi_{1}^{\prime}=\xi_{1}, \quad \xi_{2}^{\prime}=t \xi_{2}-\beta\left((1+a) \xi_{1}+a\right)
$$


Therefore $\xi_{1}$ is an invariant of the algebra $A$, and we may distinguish the following disjoint subsubcases.

Case 3.2.1. $\xi_{1}=-(1+a)^{-1} a$.

Then the second part of (12) reduces to $\xi_{2}^{\prime}=t \xi_{2}$, so we may assume either $\xi_{2}=0$ or $\xi_{2}=1$, forcing $A$ to be either as in $3.4 \mathrm{j}$ ) or $3.4 \mathrm{k}$ ), respectively. Uniqueness is clear in both cases.

Case 3.2.2. $\xi_{1} \neq-(1+a)^{-1} a$.

By (12), we are then allowed to assume $\xi_{2}=0$, whence $A$ satisfies $3.4 \mathrm{j}$ ).

Case 3.3. $y=0$.

Then $\xi_{1} \neq 0$, so we may assume $\xi_{1}=1$ by 1.14. Futhermore, for any $\beta \in k, \gamma, t \in k^{\times}$and $u, v$ as in (3.22.1), $x$ may be preplaced by

$$
\begin{aligned}
v^{t} x & =\gamma(1-\beta(a+1) \varepsilon)\left(1+t \xi_{2} \varepsilon\right) \\
& =\gamma 1+\gamma\left(t \xi_{2}-\beta(a+1)\right) \varepsilon
\end{aligned}
$$

Hence we may assume $\xi_{2}=0$ and therefore end up with $A$ as in 3.4 l).

It remains to show that the cases a) - 1) of 3.4 are mutually disjoint. This follows immediately from the fact that this is so for all the cases, subcases etc. in the preceding proof.

\section{Singular algebras}

4.0 In this section we turn to the classification problem for singular algebras in dimension two. Our first aim will be to describe the key examples. We will then proceed to derive a tight enumeration theorem before the complete classification can be carried out.

4.1 Some notation. Let $U$ be any vector space over $k$. We write $U^{*}=\operatorname{Hom}_{k}(U, k)$ for its dual space and denote by

$$
U^{*} \times U \longrightarrow k,\left(u^{*}, u\right) \longmapsto<u^{*}, u>,
$$

the canonical pairing. Recall that $U \otimes U^{*}$ identifies inside $\operatorname{End}_{k}(U)$ via the formula

$$
\left(u \otimes u^{*}\right)(v)=<u^{*}, v>u
$$

for $u, v \in U, u^{*} \in U^{*}$. In fact, we have $U \otimes U^{*}=\operatorname{End}_{k}(U)$ under this identification if and only if $U$ is finite-dimensional. Furthermore, the decomposable tensors $u \otimes u^{*}, u \in U, u^{*} \in U^{*}$ furnish precisely the elements of rank at most one in $\operatorname{End}_{k}(U)$.

4.2 The key examples. Let $\beta: V \times V \longrightarrow k$ be a bilinear form and $u \in V$. Then we define an algebra $A(u, \beta) \in \operatorname{Alg}(V)$ by the multiplication rule

$$
x A(u, \beta) y=\beta(x, y) u \quad(x, y \in V) .
$$

Clearly, $A(u, \beta)$ is bisingular in the sense that it is both left and right singular. 
On the other hand, suppose we are given elements $u^{*} \in V^{*}$ and $f \in \operatorname{End}_{k}(V)$. Then we define an algebra $A\left(u^{*}, f\right) \in \operatorname{Alg}(V)$ by

$$
x A\left(u^{*}, f\right) y=<u^{*}, y>f(x) \quad(x, y \in V) .
$$

Clearly, $A\left(u^{*}, f\right)$ is left singular. Moreover, $A$ is strictly left singular, i.e., left singular but not bisingular, if and only if $u^{*} \neq 0$ and $f \in \mathrm{GL}(V)$. In fact, if $f=v \otimes v^{*}\left(v \in V, v^{*} \in V\right)$ is a decomposable tensor in the sense of 4.1, we may combine (1), (2) with (4.1.1) to conclude

$$
A\left(u^{*}, v \otimes v^{*}\right)=A\left(v, \beta_{v^{*}, u^{*}}\right)
$$

for $v \in V, u^{*}, v^{*} \in V$, where $\beta_{v^{*}, u^{*}}: V \times V \longrightarrow k$ is the bilinear form given by

$$
\beta_{v^{*}, u^{*}}(x, y)=<v^{*}, x><u^{*}, y>\quad(x, y \in V) .
$$

Notice that $A\left(u^{*}, f\right)^{\mathrm{op}}$, for $0 \neq u^{*} \in V^{*}, f \in \mathrm{GL}(V)$, is strictly right singular in the sense that it is right singular but not bisingular. Before we can proceed to the enumeration theorem, we require the following auxiliary result.

4.3 Lemma. Notations being as in 4.1, let $U$ be a vector space over $k$ and $W \subset \operatorname{End}_{k}(U) a$ subspace whose elements have rank at most one. Then $W \subset u \otimes U^{*}$ for some $u \in U$ or $W \subset U \otimes u^{*}$ for some $u^{*} \in U^{*}$.

Proof. We may assume $W \neq(0)$ and pick a nonzero element $f \in W$, which can be written as $f=u \otimes u^{*}, 0 \neq u \in U, 0 \neq u^{*} \in U^{*}$. Representing any $g \in W$ in the same way as $g=v \otimes v^{*}, v \in$ $U, v^{*} \in U^{*}$, we claim that $u, v$ or $u^{*}, v^{*}$ are linearly dependent. To prove this, suppose $u^{*}, v^{*}$ are linearly independent. Then there exists an element $x \in U$ satisfying $\left\langle u^{*}, x\right\rangle=1,\left\langle v^{*}, x\right\rangle=0$, which implies

$$
(f+g)(x)=<u^{*}, x>u+<v^{*}, x>v=u .
$$

Hence $u \in \operatorname{im}(f+g)$, and by a similar argument $v \in \operatorname{im}(f+g)$ as well. But $f+g \in W$ has rank at monst one, forcing $u, v$ to be linearly dependent and establishing our intermediate claim. Accordingly, we have $g \in u \otimes U^{*}$ or $g \in U \otimes u^{*}$. Hence $W$ belongs to the union of these two spaces, which cannot be unless it is contained in one of them.

4.4 Tight Enumeration Theorem for Singular Algebras in Dimension Two. A twodimensional algebra $A$ over $k$ is singular if and only if it satisfies one of the following mutually exclusive conditions.

a) $A$ is the two-dimensional zero algebra.

b) There exist a nonzero element $u \in V$ and a nonzero bilinear form $\beta: V \times V \rightarrow k$ such that $A \cong A(u, \beta) ;$ in this case, $A$ is bisingular.

c) There exist a nonzero element $u^{*} \in V^{*}$ and an element $f \in \operatorname{GL}(V)$ such that $A \cong A\left(u^{*}, f\right)$; in this case, $A$ is strictly left singular.

d) There exist elements $u^{*}, f$ as in c) such that $A \cong A\left(u^{*}, f\right)^{\mathrm{op}}$; in this case, $A$ is strictly right singular. 
Proof. The final statements in b), c), d) follow immediately from 4.2. Hence all four types yield singular algebras and are mutually exclusive. Conversely, suppose $A \in \operatorname{Alg}(V)$ is singular with nontrivial multiplication. We must show that $A$ satisfies one of the conditions b), c), d). By passing to $A^{\text {op }}$ if necessary, we may assume that $A$ is left singular (1.4). Then $W=L_{A}(V) \subset \operatorname{End}_{k}(V)$, the space of left multiplications by elements of $V$ relative to $A$, satisfies the hypotheses of 4.3 , so we are left with the following two cases.

Case 1. $W \subset u \otimes V^{*}$ for some $u \in V$.

Then $u \neq 0$, and there is a linear map $\varphi: V \rightarrow V^{*}$ such that

$$
L_{A}(x)=u \otimes \varphi(x) \quad(x \in V) .
$$

Denote by $\beta: V \times V \rightarrow k$ the bilinear form corresponding to $\varphi$, so

$$
\beta(x, y)=\langle\varphi(x), y>\quad(x, y \in V) .
$$

For all $x, y \in V$, we now conclude

$$
\begin{aligned}
x A y & =L_{A}(x) y=(u \otimes \varphi(x))(y) \\
& =<\varphi(x), y>u \\
& =\beta(x, y) u
\end{aligned}
$$

Hence $A=A(u, \beta)$ satisfies condition c) since $\beta=0$ would force $A$ to be the two-dimensional zero algebra, which we have ruled out.

Case 2. $W \subset V \otimes u^{*}$ for some $u^{*} \in V^{*}$.

Then $u^{*} \neq 0$, and there is a linear map $f: V \longrightarrow V$ such that

$$
L_{A}(x)=f(x) \otimes u^{*} \quad(x \in V)
$$

We therefore obtain, for all $x, y \in V$ :

$$
\begin{aligned}
x A y & =L_{A}(x) y=\left(f(x) \otimes u^{*}\right)(y) \\
& =<u^{*}, y>f(x)
\end{aligned}
$$

Hence $A=A\left(u^{*}, f\right)$, and if $f$ is not invertible, $A$ satisfies condition b) by (4.2.3). On the other hand, for $f \in \mathrm{GL}(V), A$ satisfies condition c). This completes the proof.

4.5 We will now consider bisingular algebras more closely. To this end, let us write $\operatorname{Bif}(V)$ for the space of bilinear forms on $V$. For $\beta \in \operatorname{Bif}(V)$, we call

$$
\begin{aligned}
& \operatorname{Rad}_{1} \beta=\{x \in V \mid \beta(x, V)=(0)\}, \\
& \operatorname{Rad}_{\mathrm{r}} \beta=\{x \in V \mid \beta(V, x)=(0)\}
\end{aligned}
$$

the left, right radical, respectively, of $\beta$, so

$$
\operatorname{Rad} \beta=\left(\operatorname{Rad}_{l} \beta\right) \cap\left(\operatorname{Rad}_{\mathrm{r}} \beta\right)
$$

is its two-sided radical. 
4.6 Proposition. Suppose $u, v \in V$ and $\beta, \gamma \in \operatorname{Bif}(V)$ are all nonzero. Then for any $\varphi$ the following statements are equivalent.

(i) $\varphi: A(u, \beta) \stackrel{\sim}{\longrightarrow} A(v, \gamma)$ is an isomorphism.

(ii) $\varphi \in \mathrm{GL}(V)$, and there exists an element $a \in k^{\times}$such that

$$
\varphi(u)=a v, \gamma \circ(\varphi \times \varphi)=a \beta
$$

Proof. (i) is equivalent to $\varphi \in \mathrm{GL}(V)$ and

$$
\beta(x, y) \varphi(u)=\gamma(\varphi(x), \varphi(y)) v
$$

for all $x, y \in V$. The assertion follows from this at once.

4.7 Corollary. Suppose $u \in V$ and $\beta, \gamma \in \operatorname{Bif}(V)$ are all nonzero. Then the following statements are equivalent.

(i) $A(u, \beta) \cong A(u, \gamma)$.

(ii) There are elements $\varphi \in \mathrm{GL}(V), a \in k^{\times}$satisfying $\varphi(u)=u$ and $\gamma \circ(\varphi \times \varphi)=a \beta$.

Proof. If $\psi: A(u, \beta) \sim A(u, \gamma)$ is an isomorphism, 4.6 yields an element $b \in k^{\times}$such $\psi(u)=b u$ and $\gamma \circ(\psi \times \psi)=b \beta$. Hence $\varphi=b^{-1} \psi, a=b^{-1}$ satisfy (ii). Reading this argument backwards completes the proof.

4.8 Corollary. Suppose $u \in V$ and $\beta \in \operatorname{Bif}(V)$ are both nonzero. Then $A(u, \beta) \cong A(u, a \beta)$ for all $a \in k^{\times}$.

Proof. Setting $\gamma=a \beta, 4.7$ (ii) is fulfilled with $\varphi=\mathbf{1}$.

4.9 In order to classify bisingular algebras, we fix once and for all a basis $\left(e_{1}, e_{2}\right)$ of $V$ and identify $V=k^{2}, \operatorname{End}_{k}(V)=\operatorname{Mat}_{2}(k)$ and $\operatorname{Bif}(V)=\operatorname{Mat}_{2}(k)$ accordingly; the latter identification matches $S \in \operatorname{Mat}_{2}(k)$ with $\beta_{S} \in \operatorname{Bif}(V)$ given by

$$
\beta_{S}(x, y)={ }^{t} x S y \quad\left(x, y \in V=k^{2}\right) .
$$

This clearly implies

$$
\beta_{S} \circ(P \times P)=\beta_{t}{ }_{P S P}
$$

for all $S, P \in \operatorname{Mat}_{2}(k)$. Hence 4.7 may be rephrased as follows.

4.10 Proposition. For all nonzero elements $S, T \in \operatorname{Mat}_{2}(k)$, we have

$$
A\left(e_{1}, \beta_{S}\right) \cong A\left(e_{1}, \beta_{T}\right)
$$

if and only if there are elements $w \in k^{2}$ and $a \in k^{\times}$satisfying

$$
P=\left(e_{1}, w\right) \in \mathrm{GL}_{2}(k),{ }^{t} P T P=a S .
$$


4.11 Classification Theorem for Bisingular Algebras of Dimension Two. Notations being as in 4.9, an algebra with nontrivial multiplication is bisingular of dimension 2 if and only if it is isomorphic to $A\left(e_{1}, \beta_{S}\right)$ where $S \in$ Mat $_{2}(k)$ satisfies one of the following mutually exclusive conditions.
a) $S=\left(\begin{array}{ll}0 & 0 \\ 0 & 1\end{array}\right)$
b) $S=\left(\begin{array}{ll}0 & 0 \\ 1 & 0\end{array}\right)$.
c) $S=\left(\begin{array}{cc}0 & 1 \\ -1 & 1\end{array}\right)$.
d) $S=\left(\begin{array}{ll}0 & 1 \\ a & 0\end{array}\right)$ where $a \in k$ is unique.
e) $S=\left(\begin{array}{ll}1 & 0 \\ 0 & b\end{array}\right)$ where $b \in k$ is unique $\bmod k^{\times 2}$.
f) $S=\left(\begin{array}{ll}1 & 0 \\ 1 & b\end{array}\right)$ where $b \in k$ is unique.

Remark. The algebra of type 4.11 a) is commutative associative and, in fact, appears already in Peirce [16, p.122] under the label $\left(c_{2}\right)$. The algebra of type $4.11 \mathrm{~d}$ ) with $a=-1$ is the unique nonabelian Lie algebra of dimension two, cf. Jacobson $[9$, p. 10]. Finally, uniqueness of the parameters in d), e), f) is to be understood in the sense of the remark following 3.4.

Proof. All algebras listed in a) - f) above are bisingular with nontrivial multiplication. Conversely, let $A \in \operatorname{Alg}(V)$ be so. Then there are nonzero elements $u \in V, \beta \in \operatorname{Bif}(V)$ satisfying $A=A(u, \beta)$ $(4.2,4.4)$. We now distinguish the following cases.

Case 1. $u \in \operatorname{Rad} \beta$.

Extending $u$ to a basis $(u, v)$ of $V$, we conclude $\beta(u, u)=\beta(u, v)=\beta(v, u)=0, a=\beta(v, v) \in k^{\times}$, so 4.8 allows us to assume $a=1$, forcing $A$ to be as in 4.11 a).

Case 2. $u \in\left(\operatorname{Rad}_{1} \beta\right)-(\operatorname{Rad} \beta)$.

Here $u$ can be extended to a basis $(u, w)$ of $V$ satisfying $\beta(u, u)=\beta(u, w)=0, \beta(w, u)=1$. Setting

$$
v:=-\beta(w, w) u+w,
$$

we still have a basis $(u, v)$ of $V$ satisfying $\beta(u, u)=\beta(u, v)=0, \beta(v, u)=1$. In addition we obtain $\beta(v, v)=0$, forcing $A$ to be as in $4.11 \mathrm{~b})$.

Case 3. $u \in\left(\operatorname{Rad}_{\mathrm{r}} \beta\right)-(\operatorname{Rad} \beta)$.

Then the algebra $A^{\text {op }}$, which satisfies the conditions of Case 2, is as in $4.11 \mathrm{~b}$ ), forcing $A$ to be as in $4.11 \mathrm{~d}$ ) with $a=0$.

Case 4. $u \notin\left(\operatorname{Rad}_{l} \beta\right) \cup\left(\operatorname{Rad}_{\mathrm{r}} \beta\right)$.

Case 4.1. $\beta(u, u)=0$.

Then the linear forms $\beta(u, \cdot), \beta(\cdot, u)$ both have kernel $k u$ and hence are linearly dependent: $\beta(\cdot, u)=a \beta(u, \cdot)$ for some $a \in k^{\times}$. Hence $u$ extends to a basis $(u, w)$ of $V$ satisfying $\beta(u, w)=1$, $\beta(w, u)=a$. Replacing $w$ by $v=b u+c w(b, c \in k)$, we have $\beta(u, v)=1$ and $\beta(v, u)=a$ iff $c=1$, 
and in this case a short computation gives

$$
\beta(v, v)=(a+1) b+\beta(w, w) .
$$

Case 4.1.1. $a=-1$.

We put $d=\beta(w, w)$.

Case 4.1.1.1. $d=0$.

Then $A$ is as in $4.11 \mathrm{~d}$ ) with $a=-1$.

Case 4.1.1.2. $d \neq 0$.

Then $A \cong A\left(e_{1}, \beta_{S}\right), S=\left(\begin{array}{cc}0 & 1 \\ -1 & d\end{array}\right)$, and the computation

$$
\begin{aligned}
\left(\begin{array}{cc}
1 & 0 \\
0 & d^{-1}
\end{array}\right)\left(\begin{array}{cc}
0 & 1 \\
-1 & d
\end{array}\right)\left(\begin{array}{cc}
1 & 0 \\
0 & d^{-1}
\end{array}\right) & =\left(\begin{array}{cc}
1 & 0 \\
0 & d^{-1}
\end{array}\right)\left(\begin{array}{cc}
0 & d^{-1} \\
-1 & 1
\end{array}\right) \\
& =\left(\begin{array}{cc}
0 & d^{-1} \\
-d^{-1} & d^{-1}
\end{array}\right),
\end{aligned}
$$

combined with 4.10 , shows that $A$ is as in $4.11 \mathrm{c}$ ).

Case 4.1.2. $a \neq-1$.

Then the appropriate choice of $\mathrm{b}$ in (1) implies $\beta(v, v)=0$, forcing $A$ to be as in $4.11 \mathrm{~d}$ ).

Case 4.2. $\beta(u, u) \neq 0$.

By 4.8, we may assume $\beta(u, u)=1$.

Case 4.2.1. $\beta(u, \cdot), \beta(\cdot, u)$ are linearly dependent.

Then evaluation at $u$ yields $\beta(u, \cdot)=\beta(\cdot, u)$, and the kernel of this linear form is spanned by some $v \in V$, forcing $(u, v)$ to be a basis of $V$ and $A$ to be as in $4.11 \mathrm{e})$.

Case 4.2.2. $\beta(u, \cdot), \beta(\cdot, u)$ are linearly independent.

Then there exists a vector $v \in V$ such that $\beta(u, v)=0, \beta(v, u)=1$, forcing $A$ to be as in $4.11 \mathrm{f})$.

It remains to show that conditions a) - f) of 4.11 are mutually exclusive and that the parameters appearing in d), e), f) are unique as indicated. By 4.7, the property of $e_{1}$ to belong to the left, right, two-sided radical, respectively, of $\beta_{S}\left(S \in \mathrm{Mat}_{2}(k)\right)$ only depends on the isomorphism class of $A\left(e_{1}, \beta_{S}\right)$. The same holds true for $\beta_{S}\left(e_{1}, e_{1}\right)$ to be nonzero and the linear forms $\beta_{S}\left(e_{1}, \cdot\right), \beta_{S}\left(\cdot, e_{1}\right)$ to be linearly dependent. This proves mutual exclusiveness except possibly for cases c), d). Given $a \in k, c \in k, d \in k^{\times}$, the relation

$$
\begin{aligned}
\left(\begin{array}{ll}
1 & c \\
0 & d
\end{array}\right)\left(\begin{array}{ll}
0 & 1 \\
a & 0
\end{array}\right)\left(\begin{array}{ll}
1 & c \\
0 & d
\end{array}\right) & =\left(\begin{array}{ll}
1 & 0 \\
c & d
\end{array}\right)\left(\begin{array}{cc}
0 & d \\
a & a c
\end{array}\right) \\
& =\left(\begin{array}{cc}
0 & d \\
d a & c d(a+1)
\end{array}\right)
\end{aligned}
$$

not only settles this remaining case (4.10) but also shows that $a$ in d) is unique. Similarly, given $b \in k$, the relations

$$
\left(\begin{array}{ll}
1 & c \\
0 & d
\end{array}\right)\left(\begin{array}{ll}
1 & 0 \\
0 & b
\end{array}\right)\left(\begin{array}{ll}
1 & c \\
0 & d
\end{array}\right)=\left(\begin{array}{ll}
1 & 0 \\
c & d
\end{array}\right)\left(\begin{array}{cc}
1 & c \\
0 & b d
\end{array}\right)=\left(\begin{array}{cc}
1 & c \\
c & c^{2}+d^{2} b
\end{array}\right)
$$




$$
\begin{aligned}
{ }^{t}\left(\begin{array}{ll}
1 & c \\
0 & d
\end{array}\right)\left(\begin{array}{ll}
1 & 0 \\
1 & b
\end{array}\right)\left(\begin{array}{ll}
1 & c \\
0 & d
\end{array}\right) & =\left(\begin{array}{ll}
1 & 0 \\
c & d
\end{array}\right)\left(\begin{array}{cc}
1 & c \\
1 & c+d b
\end{array}\right) \\
& =\left(\begin{array}{cc}
1 & c \\
c+d & c^{2}+c d+d^{2} b
\end{array}\right)
\end{aligned}
$$

show that the parameters $b$ in e), f) are unique as indicated.

4.12 We finally turn to strictly left singular algebras as in $4.4 \mathrm{c}$ ). As in the bisingular case, our starting point will be an elementary isomorphism criterion, similar to 4.6. For $\varphi \in \operatorname{End}_{k}(V)$ we denote by $\varphi^{*} \in \operatorname{End}_{k}\left(V^{*}\right)$ its dual endomorphism.

4.13 Proposition. Suppose $u^{*}, v^{*} \in V^{*}$ are nonzero and $f, g \in \mathrm{GL}(V)$. Then for any $\varphi$ the following statements are equivalent.

(i) $\varphi: A\left(u^{*}, f\right) \stackrel{\sim}{\longrightarrow} A\left(v^{*}, g\right)$ is an isomorphism.

(ii) $\varphi \in \mathrm{GL}(V)$, and there exists an element $a \in k^{\times}$such that

$$
\varphi f \varphi^{-1}=a g, \varphi^{*}\left(v^{*}\right)=a u^{*} .
$$

Proof. (i) is equivalent to $\varphi \in \mathrm{GL}(V)$ and

$$
\begin{aligned}
<u^{*}, y>\varphi(f(x)) & =<v^{*}, \varphi(y)>g(\varphi(x)) \\
& =<\varphi^{*}\left(v^{*}\right), y>g(\varphi(x))
\end{aligned}
$$

for all $x, y \in V$. The assertion follows from this at once.

4.14 Corollary. Suppose $u^{*} \in V^{*}$ is nonzero and $f, g \in \mathrm{GL}(V)$. Then the following statements are equivalent.

(i) $A\left(u^{*}, f\right) \cong A\left(u^{*}, g\right)$.

(ii) There are elements $\varphi \in \mathrm{GL}(V), a \in k^{\times}$satisfying $\varphi f \varphi^{-1}=$ ag and $\varphi^{*}\left(u^{*}\right)=u^{*}$.

Proof. If $\psi: A\left(u^{*}, f\right) \stackrel{\sim}{\longrightarrow} A\left(u^{*}, g\right)$ is an isomorphism, 4.13 yields an element $a \in k^{\times}$such that $\psi f \psi^{-1}=a g, \psi^{*}\left(u^{*}\right)=a u^{*}$. Hence $\varphi=a^{-1} \psi$ and $a$ satisfy (ii). Reading this argument backwards completes the proof.

4.15 Corollary. Let $u^{*} \in V^{*}$ be nonzero and $f \in \operatorname{GL}(V)$. Then $A\left(u^{*}, f\right) \cong A\left(u^{*}\right.$, af $)$ for all $a \in k^{\times}$.

Proof. Setting $g=a f, 4.14$ (ii) is fulfilled with $\varphi=\mathbf{1}$ and $a^{-1}$ in place of $a$. 
4.16 Classification Theorem for Strictly Left Singular Algebras of Dimension Two. Fixing once and for all a nonzero element $u^{*} \in V^{*}$, a two-dimensional $k$-algebra is strictly left singular if and only if it is isomorphic to $A\left(u^{*}, f\right)$ for some $f \in \operatorname{GL}(V)$. Furthermore, given $f, g \in \mathrm{GL}(V)$, the following statements are equivalent.

(i) $A\left(u^{*}, f\right) \cong A\left(u^{*}, g\right)$.

(ii) There exists an element $a \in k^{\times}$satisfying the following conditions: $f$ and ag have the same characteristic polynomial as well as the same minimum polynomial and, for all $b \in$ $k, g^{*}\left(u^{*}\right)=b u^{*}$ if and only if $f^{*}\left(u^{*}\right)=a b u^{*}$.

Proof. The first part follows from 4.4 and 4.13. It therefore suffices to prove the equivalence of (i) and (ii).

(i) $\Rightarrow$ (ii). By 4.14 we find elements $\varphi \in \operatorname{GL}(V)$ and $a \in k^{\times}$satisfying $\varphi f \varphi^{-1}=a g$ and $\varphi^{*}\left(u^{*}\right)=u^{*}$. Hence $f$ and $a g$ have the same characteristic and minimum polynomials; in addition, $f^{*} \varphi^{*}=a \varphi^{*} g^{*}$, so for all $b \in k$ the relation $g^{*}\left(u^{*}\right)=b u^{*}$ implies $f^{*}\left(u^{*}\right)=a b u^{*}$ and conversely.

(ii) $\Rightarrow$ (i). By 4.15 we may assume $a=1$. Then we distinguish the following cases.

Case 1. $u^{*}$ and $g^{*}\left(u^{*}\right)$ are linearly independent.

Let $\varphi \in \operatorname{End}_{k}(V)$ be given by $\varphi^{*}\left(u^{*}\right)=u^{*}, \varphi^{*}\left(g^{*}\left(u^{*}\right)\right)=f^{*}\left(u^{*}\right)$. Since $u^{*}$ and $f^{*}\left(u^{*}\right)$ are linearly independent as well by hypothesis, we actually have $\varphi \in \mathrm{GL}(V)$. In order to show $\varphi^{*} g^{*}=f^{*} \varphi^{*}$, it suffices to prove that both sides agree on the basis $u^{*}, g^{*}\left(u^{*}\right)$ of $V^{*}$. This is obvious for $u^{*}$ whereas for $g^{*}\left(u^{*}\right)$, using the fact that $f^{*}, g^{*}$ have the same characteristic polynomial, one may argue as follows:

$$
\begin{aligned}
\varphi^{*} g^{*}\left(g^{*}\left(u^{*}\right)\right) & =\varphi^{*} g^{* 2}\left(u^{*}\right) \\
& =\varphi^{*}\left(\left(\operatorname{trace} g^{*}\right) g^{*}\left(u^{*}\right)-\left(\operatorname{det} g^{*}\right) u^{*}\right) \\
& =\left(\operatorname{trace} f^{*}\right) f^{*}\left(u^{*}\right)-\left(\operatorname{det} f^{*}\right) u^{*} \\
& =f^{* 2}\left(u^{*}\right)=f^{*} \varphi^{*}\left(g^{*}\left(u^{*}\right)\right) .
\end{aligned}
$$

Thus $\varphi^{*} g^{*}=f^{*} \varphi^{*}$, which implies $\varphi f \varphi^{-1}=g$, hence $A\left(u^{*}, f\right) \cong A\left(u^{*}, g\right)$ by 4.14 .

Case 2. $g^{*}\left(u^{*}\right)=b u^{*}$ for some $b \in k$.

Then (ii) gives

$$
f^{*}\left(u^{*}\right)=b u^{*}
$$

as well. Also, since $f$ and $g$ have the same characteristic and minimum polynomials, it is well known and easily seen that they are conjugate under (inner) automorphisms: There exists an element $\varphi \in \mathrm{GL}(V)$ satisfying $\varphi f \varphi^{-1}=g$. This implies $\varphi^{*} g^{*}=f^{*} \varphi^{*}$, hence

$$
f^{*}\left(\varphi^{*}\left(u^{*}\right)\right)=b \varphi^{*}\left(u^{*}\right)
$$

If $u^{*}, \varphi^{*}\left(u^{*}\right)$ are linearly independent, (1), (2) imply $f=b \mathbf{1}=g$, and (i) follows. On the other hand, if $u^{*}, \varphi^{*}\left(u^{*}\right)$ are linearly dependent, we conclude $\varphi^{*}\left(u^{*}\right)=c u^{*}$ for some $c \in k^{\times}$, so the dual of $\psi=c^{-1} \varphi$ fixes $u^{*}$ while we still have $\psi f \psi^{-1}=g$. Hence (i) follows again (4.14), and the proof of the theorem is complete.

4.17 Reading 4.16 for $A^{\text {op }}$ instead of $A$, one arrives at a classification theorem for strictly right singular algebras of dimension two. Details will be omitted. 


\section{Quasi-composition algebras}

5.0 In this section, we connect our preceding results with the author's classification of twodimensional quasi-composition algebras [14, Proposition 2]. This will not only lead to sharper conclusions but also allows base fields of characteristic two. In addition, we will be able to by-pass a somewhat sticky point in the proof of the original classification result. ${ }^{1}$

5.1 Quasi-composition algebras revisited. Following [14], a quasi-composition algebra over $k$ is a $k$-algebra $A$ such that there exists a quadratic form $n: A \longrightarrow k$ satisfying the following conditions: The symmetric bilinear form induced by $n$ is nondegenerate, and $n$ permits composition, i.e, $n(x y)=n(x) n(y)$ for all $x, y \in A$. Hence ordinary composition algebras are quasi-composition algebras containing a unit.

\subsection{Proposition.}

a) Quasi-composition algebras of finite dimension are regular.

b) The unital heart of a two-dimensional quasi-composition algebra is étale.

c) Let $K \in \operatorname{Al} g(V)$ be a two-dimensional étale $k$-algebra, with norm $n$, and $f, g \in \mathrm{GL}(V)$. For $K^{(f, g)}$ to be a quasi-composition algebra it is necessary and sufficient that $f, g$ be similarity transformations of the quadratic space $(V, n)$.

Proof. a) Given a quasi-composition algebra $A$ over $k$, let $n$ be as in 5.1 and suppose $x \in A$ is anisotropic relative to $n$. Since $n$ permits composition, $L_{A}(x)$ is a similarity transformation relative to $n$, hence bijective. This proves left regularity. Right regularity is established the same way.

b) This is an immediate and well known consequence of Kaplansky's trick 1.5 in its classical form [10], phrased in the terminology of the Unital Heart Proposition 1.10.

c) Suppose first that $f, g$ are similarity transformations of $(V, n): n \circ f=a n, n \circ g=b n$ for some $a, b \in k^{\times}$. Then $a b n$ permits composition for the algebra $K^{(f, g)}$, which is therefore a quasicomposition algebra. Conversely, let this be so. Then Kaplansky's trick in its classical form [10] combined with 1.10 shows $K^{(f, g)} \cong K^{\left(f^{\prime}, g^{\prime}\right)}$ where $f^{\prime}, g^{\prime}$ are orthogonal transformations of $(V, n)$. By the Isomorphism Criterion 1.12, we find elements $u, v \in K^{\times}, \sigma \in \operatorname{Aut}(K)$ satisfying $f=L\left(v^{-1}\right) \sigma f^{\prime} \sigma^{-1} L(u v), g=L\left(u^{-1}\right) \sigma g^{\prime} \sigma^{-1} L(u v)$. Hence $f, g$ preserve $n$ up to nonzero scalars, and we are done.

5.3 Lemma. Let $K \in \operatorname{Alg}(V)$ be an étale k-algebra of dimension two and $n$ its norm. For $f \in \operatorname{End}_{k}(V)$ to be a similarity transformation of $(V, n)$ it is necessary and sufficient that there exist elements $u \in K^{\times}, \sigma \in \operatorname{Aut}(K)$ satisfying $f=L(u) \sigma$.

Proof. The condition is clearly sufficient. Conversely suppose $f$ is a similarity transformation of $(V, n)$. Then $u=f(1) \in K^{\times}$, and $L\left(u^{-1}\right) f$ is orthogonal relative to $n$. It also fixes 1 , hence is an automorphism of $K$.

5.4 Classification Theorem for Two-dimensional Quasi-Composition Algebras. Let $A$ be a two-dimensional quasi-composition algebra over $k$ with unital heart $K$. Then $A$ is isomorphic to precisely one of the following.

\footnotetext{
${ }^{1}$ In the argument following $\left[14,\left(3^{\prime}\right)\right.$, p. 217] it is tacitly assumed that the automorphism $\varphi^{\prime}$ does not depend on $x$. A justification of this assumption is possible but awkward.
} 
a) $K$.

b) $K^{(\tau, \mathbf{1})}$.

c) $K^{(\mathbf{1}, \tau)}$.

d) $K^{(\tau, L(y) \tau)}, y \in S(K)$.

Furthermore, given $y, z \in S(K)$, we have

$$
K^{(\tau, L(y) \tau)} \cong K^{(\tau, L(z) \tau)}
$$

if and only if $y \equiv z \bmod S(K)^{3}$ or $y \equiv{ }^{\tau} z \bmod S(K)^{3}$.

Proof. The unital heart of $A$ being étale (5.2 b)), A fits into the Tight Enumeration Theorem 2.3, where all types a) - g) must be excluded whose exponents do not consist of similarity transformations relative to $n(5.2 \mathrm{c}))$. Combining the decomposition $(2.2 .1)$ with 5.3 , we see that precisely the types b), c), d) (with $y=0$ ) remain. This proves the first part of the theorem. The second part is simply a restatement of $2.12 \mathrm{c}$ ).

\section{Acknowledgements}

A substantial part of this work was carried out while the author was visiting the Department of Mathematics of the University of Oviedo. He would like to express his gratitude to J. Anquela and T. Cortes for their hospitality and lively discussions. Thanks are also due to R. Börger, O. Loos, C. Martinez, D. Pumplün, H. Röhrl, S. Walcher and, in particular, to M.L. Racine for valuable comments.

\section{References}

1. Albert, A. A. Nonassociative algebras. Ann. of Math. (2) 43 (1942), 685 - 707.

2. Althoen, S. C. and Hansen, K.D. Two-dimensional real algebras with zero divisors. Acta Sci. Math. (Sceged) 56 (1992), $23-42$.

3. Althoen, S. C. and Kugler, L.D. When is $\mathbf{R}^{2}$ a division algebra? Amer. Math. Monthly 90 (1983), 625 - 635.

4. Bourbaki, N. "Algebra I". Addison-Wesley: Reading, MS, USA, 1974.

5. Burdujan, I. Types of nonisomorphic two-dimensional real division algebras. Proceedings of the national conference on algebra (Romanian) (la/c si, 1984). An. /c Stiin/c t. Univ. "al. I. Cuza" Ia/c si Sec/c t. I a Mat. (N.S.) 31 (1985), 102 - 105.

6. Cali, A.L. and Josephy, M. Two-dimensional real division algebras. Rev. Un. Mat. Argentina 32 (1985), 58 - 63.

7. Elduque, A. and Pérez, J.M. Infinite dimensional quadratic forms admitting composition. Proc. Amer. Math. Soc. 125 (1997), 2207 - 2216.

8. Gottschling, E. Die zweidimensionalen reellen Divisionsalgebren. Seminarberichte des Fachbereichs Mathematik der FernUniversität-GHS in Hagen 63 (1998), 228 - 261.

9. Jacobson, N. "Lie algebras." Interscience: New York, 1962.

10. Kaplansky, I. Infinite-dimensional quadratic forms permitting composition. Proc. Amer. Math. Soc. 4 (1953), 956 - 960 .

11. Koecher, M. Über Standardkonstruktionen von nicht-assoziativen Algebren. Sitzungsber. Bayer. Akad. Wiss. Math.-Natur. Kl. 3 (1974), 35 - 57. 
12. Luchian, T. A classification of linear algebras of order 2, with divisors of zero. An Stiint. Univ. "Al. I Cuza" Iasi Sect. I.IV (1958), 21 - 37.

13. Markus, L. Quadratic differential equations and nonassociative algebras. Contributions to the theory of nonlinear oscillations, Princeton, 1960, pp. 185 - 213.

14. Petersson, H.P. Quasi-composition algebras. Abh. Math. Sem. Univ. Hamburg 35 (1971), 215 - 222.

15. - Composition algebras over algebraic curves of genus zero. Trans. Amer. Math. Soc. 337 (1993), 473 493.

16. Peirce, B. Linear associative algebras. Amer. J. Math. 4 (1881), 97 - 215, addenda: 216 - 229.

17. Segre, B. La teoria della algebre ed alcune questione di realta. Rend. Mat. e Appl. serie 5, 13 (1954-5), 157 - 188.

18. Walcher, W. On algebras of rank three. Comm. Algebra 27 (1999), 3401-3438.

Holger P. Petersson

Fachbereich Mathematik

FernUniversität - Gesamthochschule in Hagen

D - 58084 Hagen

Germany

e-mail: Holger.Petersson@FernUni-Hagen.de

Eingegangen am 05. August 1999 